\title{
Tetraspanin CD37 protects against the development of B cell lymphoma
}

\author{
Charlotte M. de Winde, ${ }^{1}$ Sharon Veenbergen, ${ }^{1}$ Ken H. Young, ${ }^{2}$ Zijun Y. Xu-Monette, ${ }^{2}$ Xiao-xiao Wang, ${ }^{2}$ Yi Xia, ${ }^{2}$ Kausar J. Jabbar, ${ }^{2}$ \\ Michiel van den Brand, ${ }^{3}$ Alie van der Schaaf, ${ }^{1}$ Suraya Elfrink, ${ }^{1}$ Inge S. van Houdt, ${ }^{1}$ Marion J. Gijbels, ${ }^{4,5,6}$ Fons A.J. van de Loo, ${ }^{7}$ \\ Miranda B. Bennink, ${ }^{7}$ Konnie M. Hebeda, ${ }^{3}$ Patricia J.T.A. Groenen, ${ }^{3}$ J. Han van Krieken, ${ }^{3}$ Carl G. Figdor, ${ }^{1}$ \\ and Annemiek B. van Spriel ${ }^{1}$ \\ 'Department of Tumor Immunology, Radboud Institute for Molecular Life Sciences, Radboud University Medical Center, Nijmegen, Netherlands. ${ }^{2}$ Department of Hematopathology, The University of Texas \\ MD Anderson Cancer Center, Houston, Texas, USA. ${ }^{3}$ Department of Pathology, Radboud University Medical Center, Nijmegen, Netherlands. ${ }^{4}$ Department of Pathology and ${ }^{5}$ Department of Molecular Cenetics, \\ Cardiovascular Research Institute, Maastricht, Netherlands. ${ }^{6}$ Department of Medical Biochemistry, Academic Medical Center, Amsterdam, Netherlands. ${ }^{7}$ Department of Rheumatology, \\ Radboud University Medical Center, Nijmegen, Netherlands.
}

\begin{abstract}
Worldwide, B cell non-Hodgkin lymphoma is the most common hematological malignancy and represents a substantial clinical problem. The molecular events that lead to B cell lymphoma are only partially defined. Here, we have provided evidence that deficiency of tetraspanin superfamily member CD37, which is important for B cell function, induces the development of B cell lymphoma. Mice lacking CD37 developed germinal center-derived B cell lymphoma in lymph nodes and spleens with a higher incidence than Bc/2 transgenic mice. We discovered that CD37 interacts with suppressor of cytokine signaling 3 (SOCS3); therefore, absence of CD37 drives tumor development through constitutive activation of the IL-6 signaling pathway. Moreover, animals deficient for both $\mathrm{Cd} 37$ and $/ 16$ were fully protected against lymphoma development, confirming the involvement of the IL- 6 pathway in driving tumorigenesis. Loss of CD37 on neoplastic cells in patients with diffuse large B cell lymphoma (DLBCL) directly correlated with activation of the IL-6 signaling pathway and with worse progressionfree and overall survival. Together, this study identifies CD37 as a tumor suppressor that directly protects against B cell lymphomagenesis and provides a strong rationale for blocking the IL-6 pathway in patients with CD37- B cell malignancies as a possible therapeutic intervention.
\end{abstract}

\section{Introduction}

The majority of B cell lymphomas originate from germinal center-derived (GC-derived) B cells, which is the result of genetic defects during VDJ recombination, somatic hypermutation and class-switching recombination (1). The best-known chromosomal aberration in follicular lymphoma (FL) and diffuse large B cell lymphoma (DLBCL) is translocation of $t(14 ; 18)$, resulting in constitutive expression of BCL-2 and defective apoptosis (2), which is correlated with worse survival in patients with DLBCL (3). However, $\mathrm{t}(14 ; 18)$ can also be detected in B cells of healthy individuals, suggesting that the translocation by itself is insufficient and other genetic alterations are required to induce B cell lymphoma $(4,5)$. Detailed genomic analyses revealed the complexity of different pathways that are recurrently altered in lymphomas, including $\mathrm{B}$ cell receptor, Toll-like receptor, Notch, and NF- $\mathrm{kB}$ signaling pathways $(6,7)$. The challenge is to identify the driver mutations of these altered pathways in order to unravel how these genetic aberrations contribute to $\mathrm{B}$ cell lymphomagenesis.

IL-6, originally identified as a B cell-differentiating factor, contributes to the growth of many types of cancer, including hemato-

Authorship note: C.M. de Winde and S. Veenbergen are co-first authors. Conflict of interest: The authors have declared that no conflict of interest exists. Submitted: October 9, 2015; Accepted: December 3, 2015.

Reference information: / Clin Invest. 2016;126(2):653-666. doi:10.1172/JCI81041 logical tumors $(8,9)$. IL-6 exerts its biological function via a receptor complex composed of the IL- 6 receptor $\alpha$ chain (IL-6R $\alpha$ ) and the common signaling receptor gp130 (10) that together activate 3 pathways: the JAK/STAT3 (11), PI3K/AKT (12), and Ras/MAPK pathways (13). Activation of the IL-6 signaling pathway is negatively regulated by suppressor of cytokine signaling 3 (SOCS3), which is transcribed upon DNA binding of STAT3 homodimers (14). Cytosolic SOCS3 translocates to the plasma membrane, in which its SH2 domain binds gp130 to prevent binding and phosphorylation of STAT3 proteins $(15,16)$. Simultaneously, SOCS3 binds JAK1/2 with its kinase-inhibitory region, which targets these proteins for ubiquitination (17). In B cell lymphoma, proteins of the JAK/STAT3 signaling pathway are frequently overexpressed, contributing to cancer development and progression $(18,19)$. In addition, autocrine IL- 6 production in DLBCL provides proliferative and antiapoptotic signals, and IL-6 levels in serum correlate with the prognosis of the disease (20). To date, the underlying mechanism responsible for the constitutive activation of the IL-6 pathway in cancer is largely unknown.

Tetraspanins belong to the superfamily of transmembrane 4 proteins that form multimolecular complexes with other tetraspanin proteins, integrins, growth factors, and signaling molecules (21-24). Targeting of CD37 is currently under investigation in clinical trials for patients with B cell malignancies, but the molecular pathways have not been fully resolved $(25,26)$. CD37 is 
A

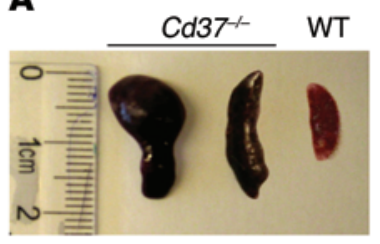

C
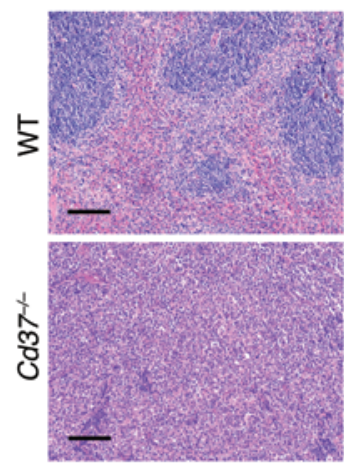

D

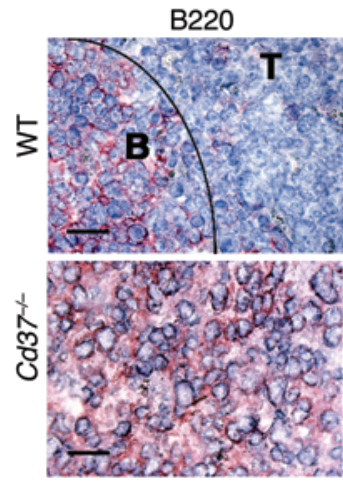

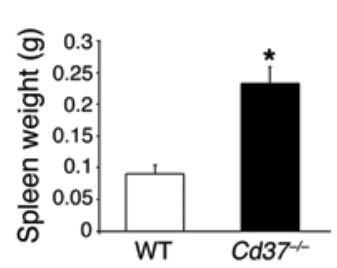

B

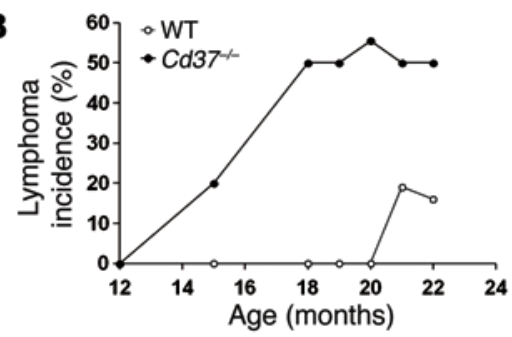

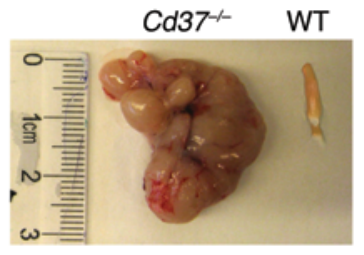
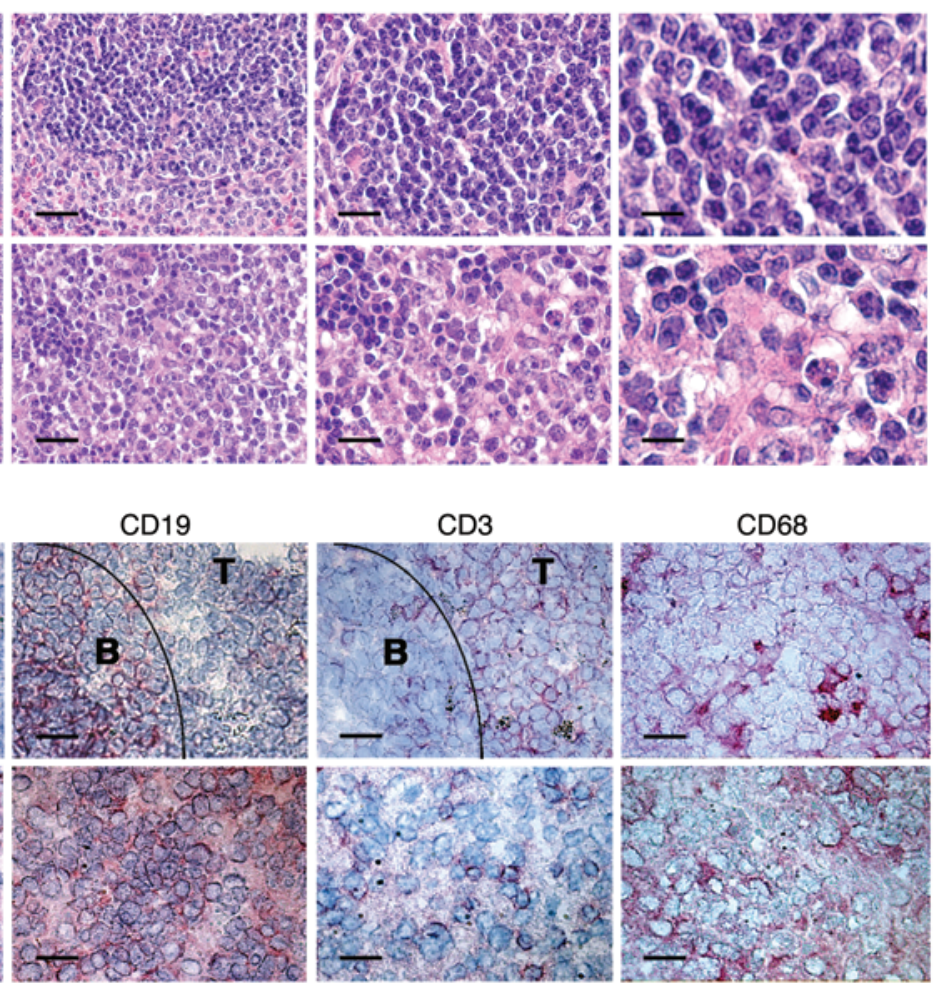

Figure 1. Spontaneous development of B cell lymphoma in Cd37-1- mice. (A) $C d 37^{-1-}$ mice demonstrate splenomegaly $(0.09 \pm 0.01 \mathrm{vs}$. $0.21 \pm 0.04 \mathrm{~g}$ spleen weight in WT and $C d 37^{-1-}$ mice, respectively; $n=10,{ }^{*} P<0.02$, unpaired 2-tailed Student's $t$ test). Data represent mean \pm SEM. (B) $C d 37^{-1-}$ mice develop large malignancies in mLNs upon aging. Macroscopic incidence of lymphomas in Cd37-/- mice $(n=48)$ versus that in age-matched WT littermates $(n=52)$.

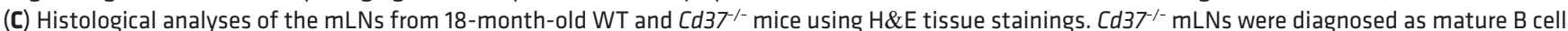
Iymphomas. Scale bar: $300 \mu \mathrm{m}$ (first column); $80 \mu \mathrm{m}$ (second column); $40 \mu \mathrm{m}$ (third column); $20 \mu \mathrm{m}$ (fourth column). (D) Immunohistochemical analyses of mLNs from 18-month-old WT and Cd37-/- mice. Tissues were stained for B220, CD19, CD3, and CD68 using fast red and hematoxylin counterstaining ( $F 4 / 80, \mathrm{Gr}, \mathrm{CD} 138$, and isotype control stainings are presented in Supplemental Figure $1 \mathrm{~A}$ ). Note the disorganization of $C d 37^{-1-} \mathrm{mLN}$ tissues that consist of small and large pleiomorphic B cells and presence of infiltrating T cells, histocytes, and macrophages. Black lines indicate the border between the B cell follicle and T cell area in WT tissues. Scale bar: $25 \mu \mathrm{m}$. Experiments were repeated 5 times with at least 4 mice of each genotype per group.

highly expressed on mature B cells and is required for optimal GC function and long-lived antibody production $(27,28)$. Mice deficient for CD37 (Cd37-- mice) have impaired humoral and cellular immune responses (29-31). This paper provides the first evidence to our knowledge that CD37 is a novel tumor suppressor that acts to suppress IL-6-driven B cell transformation in vivo.

\section{Results}

CD37 deficiency predisposes mice to develop spontaneous B cell lymphoma. CD37 is highly expressed on mature B cells and plays a fundamental role in B cell function and humoral immunity (27, 28). Although $C d 37^{-/}$mice develop normally, with unaltered numbers of lymphoid and myeloid cells in lymphoid organs (32), we observed that $C d 37^{-/}$mice became diseased during aging. By 15 months of age, $\mathrm{Cd} 37^{-/-}$mice spontaneously developed large neoplasms in mesenteric lymph nodes (mLNs), spleens, and livers in contrast to age-matched $C d 37^{+/+}$WT littermates (referred to herein as WT mice; Figure 1, A and B). Extensive analyses of mice at different ages revealed that over $50 \%$ of $C d 37^{-/}$mice developed tumors starting at 12 months. In contrast, only approximately $15 \%$ of WT mice developed tumors at the age of 22 months (Figure 1B and Supplemental Table 1; supplemental material available online with this article; doi:10.1172/JCI81041DS1), which is in line with the reported tumor occurrence in aged inbred C57BL/6 mice (33, 34). $C d 37^{+/}$heterozygous mice were healthy and did not develop lymphomas until 20 months of age with the same incidence as WT mice (data not shown). The neoplasms in Cd37/- mice were characterized by nodular growth of small and large pleiomorphic B cells, 
A

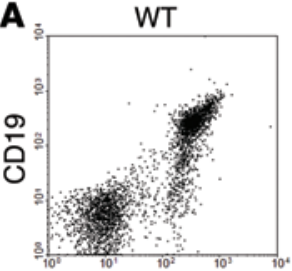

B220

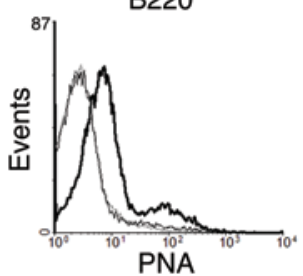

B
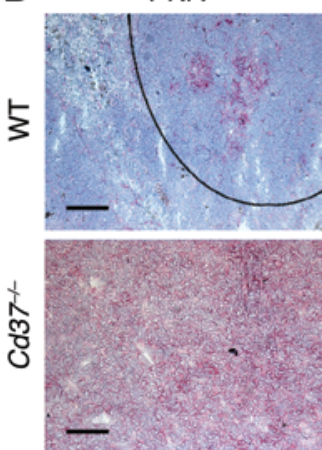

C

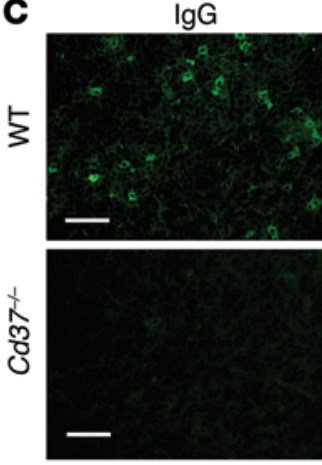

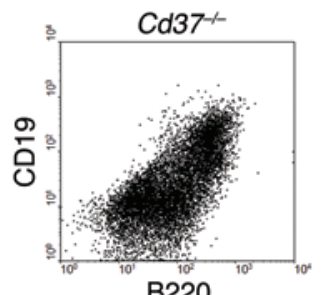

B220

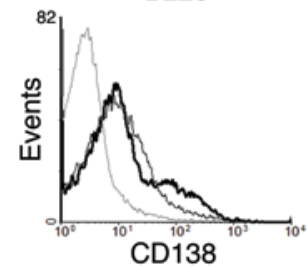

PNA
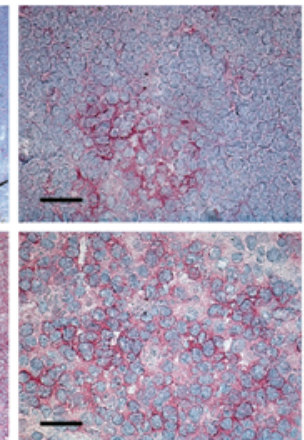

$\lg M$

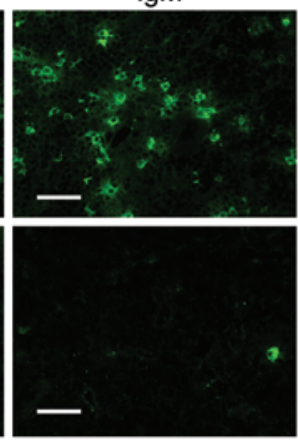

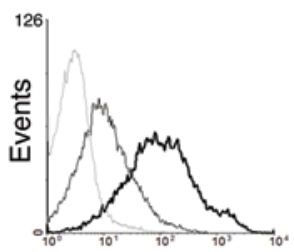

CD86

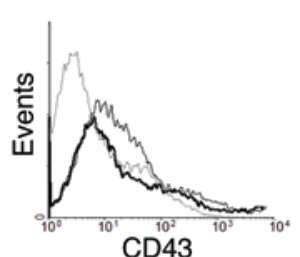

GL7
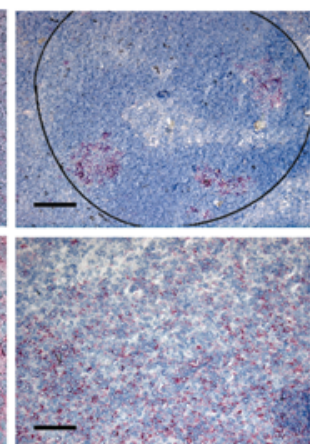

$\lg A$

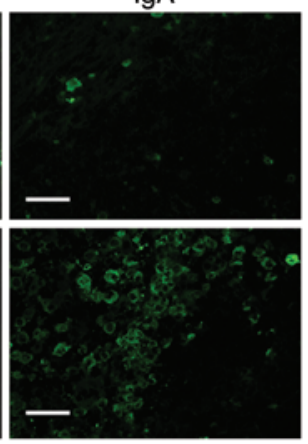

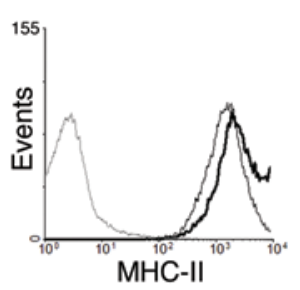

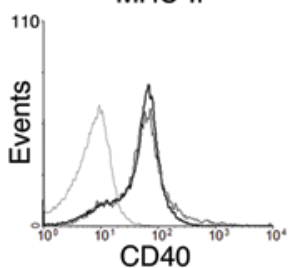

GL7

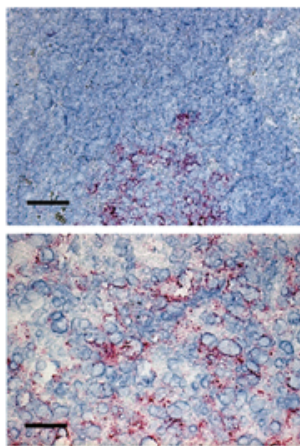

$\lg A$

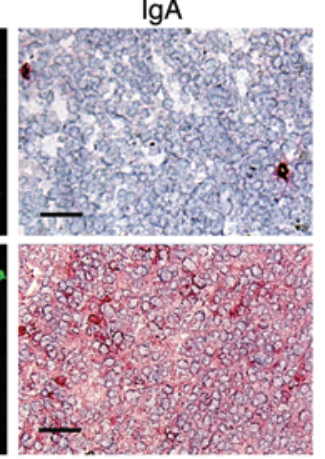

Figure 2. Phenotypical characterization of the $\mathrm{Cd}_{37^{-/}}$B cell lymphomas. (A) Flow cytometry analyses of B cells of mLNs from 18-month-old WT and Cd $37^{-/-}$mice. B cells were identified by gating on $\mathrm{CD} 19+\mathrm{B} 22 \mathrm{O}^{+}$cells that were stained for CD86, MHC-II, PNA, CD138, CD43, and CD40 (thick black lines indicate $\mathrm{Cd}_{37^{-1-}}$; thin black lines indicate $\mathrm{WT}$; gray lines indicate isotype control). (B) Immunohistochemical analyses of $\mathrm{mLNs}$ from 18-month-old WT and $\mathrm{Cd} 37^{-1-}$ mice using GC-specific staining with PNA and GL7 antibody. Scale bar: $300 \mu \mathrm{m}$ (first column); $50 \mu \mathrm{m}$ (second column); $300 \mu \mathrm{m}$ (third column); $50 \mu \mathrm{m}$ (fourth column). Lines indicate the border of the $B$ cell follicle. (C) Characterization of the $B$ cell receptor (immunoglobulin) isotype on $B$ cells from mLNs of 18-month-old WT and $\mathrm{Cd} 37^{-/-}$mice. CD19+B $22 \mathrm{O}^{+}$cells were stained for IgM, IgG, and IgA, followed by immunofluorescence (first 3 columns) and immunohistochemistry (fourth column). Scale bar: $70 \mu \mathrm{m}$. Experiments were performed 4 times with at least 4 mice of each genotype per group.

the presence of some mitotic figures, histocytes, multinucleated giant cells, and focal necrosis (Figure 1C). Immunohistochemical analyses demonstrated that the lymphomas were strongly positive for both B cell markers, B220 and CD19 (Figure 1D). CD3 ${ }^{+}$T cells and $\mathrm{CD}^{2} 8^{+}$macrophages were apparent (Figure 1D), whereas $\mathrm{Gr}^{+}$ myeloid cells and CD138 $8^{+}$plasma cells were scarce (Supplemental Figure 1A). Quantification of different immune cell subsets in Cd37-/- lymphomas and WT mLN control tissues by flow cytometry confirmed these results (Supplemental Figure 2). These data demonstrate that $C d 37$ deficiency promotes spontaneous development of B cell lymphoma in vivo.

Neoplastic cells are IgA+ GC-derived tumor cells. Expression of costimulatory molecules (CD86, CD40), MHC-II, PNA, and CD43 revealed that the lymphoma cells phenotypically resembled mature GC B cells (Figure 2A). This was confirmed by high expression of the GC markers PNA and GL7 in lymphomas of aged $C d 37^{-1-}$ mice (Figure 2B). Next, we characterized the immunoglobulin phe- notype of the mature lymphoma cells by flow cytometry. $C d 37^{-1-}$ lymphomas were all negative for $\operatorname{IgD}$, IgM, and IgG, whereas IgA was the most abundant immunoglobulin expressed by the neoplastic cells (Figure 2C and Supplemental Figure 1B). This was in contrast to the few lymphomas that were found in approximately $15 \%$ of aged WT mice, which were of the IgG or IgM phenotype (Supplemental Figure 3). Taken together, these data demonstrate that the $C d 37^{-/}$neoplasms consist of mature isotype-switched $\operatorname{IgA}^{+} \mathrm{B}$ cells that originate from the GC.

Neoplastic Cd37-/ cells are of monoclonal origin and induce disease upon transfer into young mice. To investigate the clonality status of the neoplastic $C d 37^{-/-}$cells, molecular genetic analysis of the B cell receptor was adapted using fresh lymphoma tissues from $C d 37^{-/}$mice and $\mathrm{mLN}$ tissue samples from aged WT mice as a control. In normal $\mathrm{mLN}$ tissue from WT mice, VJ gene rearrangements of the immunoglobulin heavy chain (IGH) locus demonstrated a Gaussian-like distribution of peaks, which was the result 
A
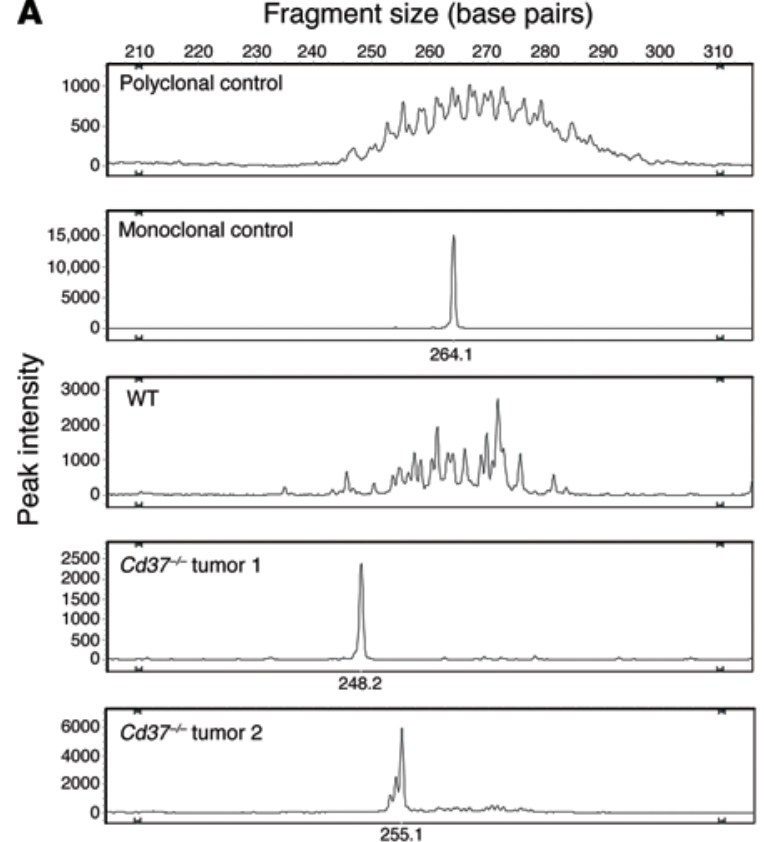

D
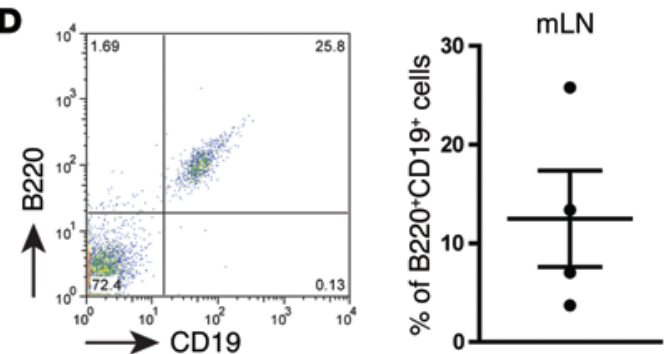
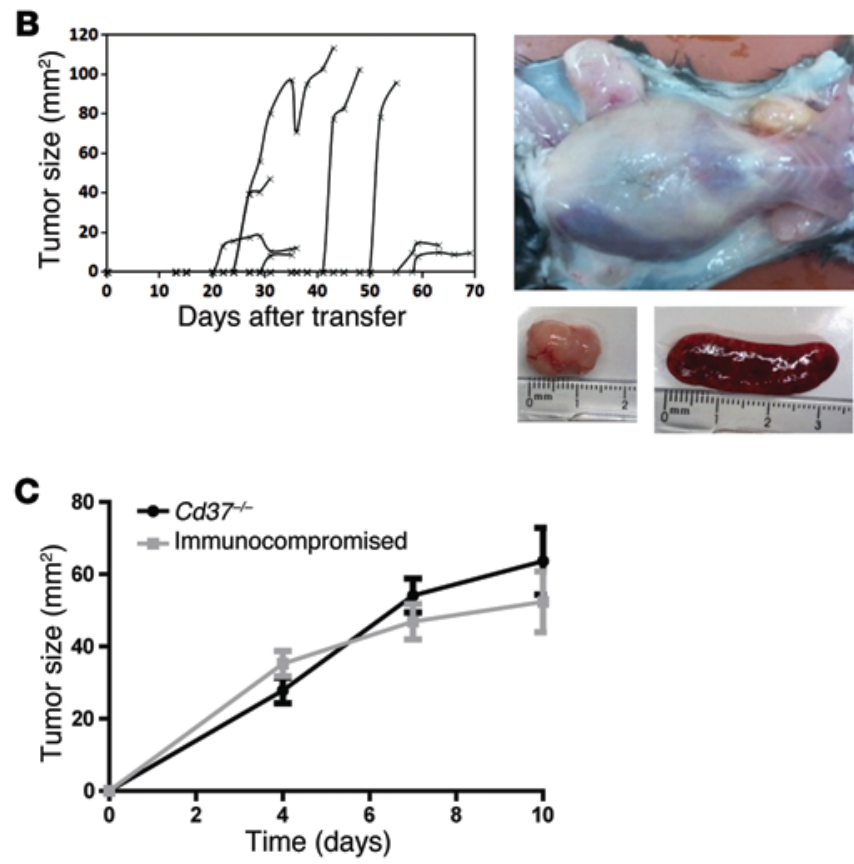

E
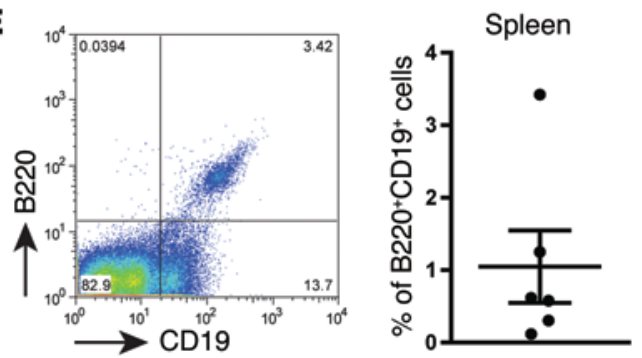

Figure 3. Clonality of Cd37-/- B cell lymphomas and adoptive transfer to young mice. (A) DNA was extracted from mLNs of 18-month-old WT and Cd37-/mice. VJ joinings from immunoglobulin heavy chain genes were amplified by the BIOMED-2 multiplex PCRs for ICH-V) rearrangements, showing FR2 (FR1 and FR3 are shown in Supplemental Figure 4). Experiments were performed 2 times with 3 mice of each genotype. Data are shown for two lymphomas and one WT control tissue as well as human polyclonal and monoclonal control samples. (B) Lymphoma cells $\left(5 \times 10^{6}\right.$ cells) were injected subcutaneously in the flanks of 6- to 8-week-old Cd37-1- mice $(n=12)$, and tumor growth was measured in time. Each line represents an individual mouse. When tumors reached $>2 \mathrm{~cm}^{2}$ in size, mice were sacrificed and lymphoid tissues were analyzed macroscopically by flow cytometry, histology, and immunohistochemistry (Supplemental Figure 5). (C) Lymphoma cells $\left(5 \times 10^{6}\right.$ cells) were injected subcutaneously in the flanks of 6- to 8 -week-old Cd37//- mice or immunocompromised nude mice ( $n=6$ mice per group), and tumor growth was measured in time. Data represent mean \pm SEM. (D and E) Lymphoma cells $\left(5 \times 10^{6}\right.$ cells) were injected intravenously in the tail veins of 6- to 8-week-old immunocompromised NSG mice $(n=6)$. These mice lack the lymphocyte compartment, including $C D 19^{+} B 220^{+} B$ cells. Mice were sacrificed after 11 days, and (D) $\mathrm{mLNs}$ and (E) spleens were analyzed for presence of $C D 19^{+} B 220^{+} B$ cells by flow cytometry (left). In the right panel, each dot represents the percentage of $C D 19+B 220^{+} B$ cells in the (D) $\mathrm{mLN}$ or (E) spleen per mouse. Data represent mean \pm SEM.

of the polyclonal nature of B cells. In contrast, $C d 37^{-/} \mathrm{B}$ cell lymphomas showed a single dominant peak, indicating a clear clonal IGH rearrangement in all framework regions (referred to herein as FR1, FR2, FR3), indicative of B cell lymphoma (Figure 3A and Supplemental Figure 4). Next, adoptive transfer experiments were performed to explore whether freshly isolated B cell lymphoma cells could be transferred into young (6- to 8-week-old) $C d 37^{-/}$mice. Transfer of fresh neoplastic cells from aged $C d 37^{-1-}$ mice rapidly induced tumor growth in healthy recipient $C d 37 /-$ mice irrespective of their age (Figure 3B). The lymphoma cells showed clear metastatic capacity, as several tumor deposits were found in secondary lymphoid organs, including spleen, inguinal, brachial, and axillary lymph nodes. Predominant expression of CD19 and B220 as well as high expression of GL7, CD86, MHCII, CD43, PNA, and IgA confirmed that all neoplasms resembled mature isotype-switched $\operatorname{IgA}^{+} \mathrm{B}$ cells of $\mathrm{GC}$ origin and showed a phenotype similar to that of the original transferred tumor (Supplemental Figure 5). Next, neoplastic cells from aged $C d 37^{-/}$mice were transferred subcutaneously or intravenously into young WT and immunocompromised mice that lack the lymphocyte compartment, including $\mathrm{CD} 19^{+} \mathrm{B}^{2} 2 \mathrm{O}^{+} \mathrm{B}$ cells. Whereas tumor cells did not grow in WT mice (data not shown), evident lymphoma growth was observed in the immunocompromised mice, similar to that in young $C d 37^{-1}$ mice after subcutaneous injection (Figure 3C). Similarly, $C d 37^{-/}$neoplastic $\mathrm{CD} 19^{+} \mathrm{B} 22 \mathrm{O}^{+} \mathrm{B}$ cells were identified in mLNs (Figure 3D) and spleens (Figure 3E) of immunocompromised mice after intravenous adoptive transfer. Thus, the neoplastic cells derived from aged $C d 3^{-/}$mice are of monoclonal origin and can induce disease and exhibit metastatic capacity upon adoptive transfer. 
A

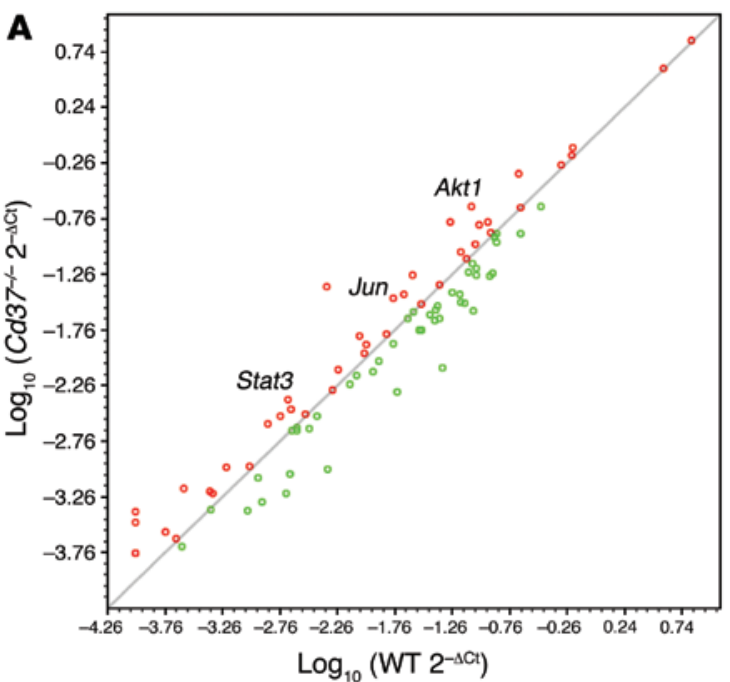

C

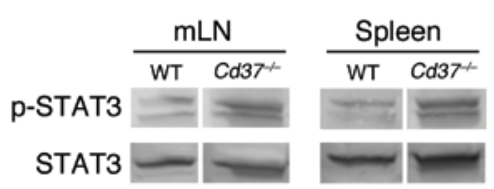

B

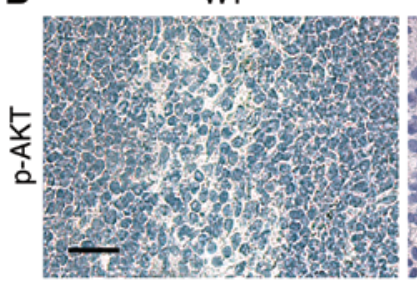

WT
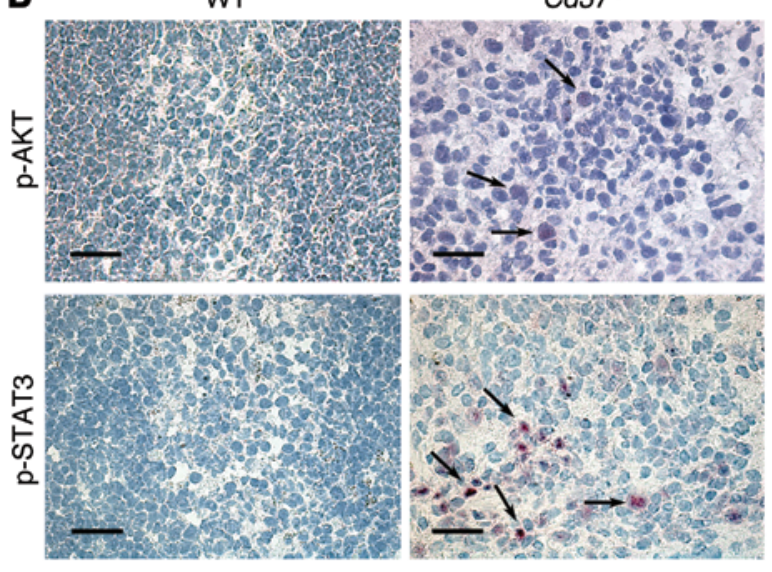

D

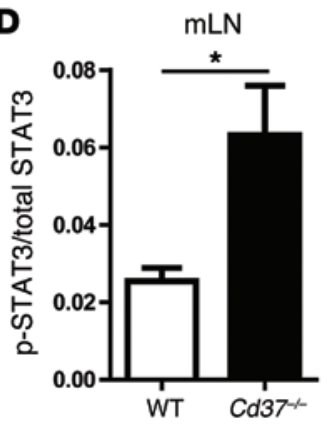

Spleen

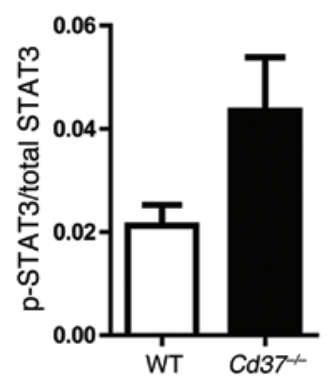

Figure 4. Constitutive activation of the IL-6 pathway in Cd37/- B cell lymphomas. (A) Cancer gene profiling was performed as described in the Methods. Among the 84 cancer-associated genes, 6 genes were upregulated and 7 genes were downregulated by approximately 2 -fold in neoplastic tissue compared with control $\mathrm{mLN}$ tissue (see also Supplemental Table 2). Red dots represent genes that were upregulated, and green dots represent genes that were downregulated. (B) Immunohistochemistry analysis of p-AKT and p-STAT3 in mLNs from 18-month-old WT and Cd37-/- mice. Scale bar: 50 um. Experiments were performed 3 times with 4 mice per group. (C and $\mathbf{D})$ Protein levels of p-STAT3 (76-78 kDa) in mLN and spleen cells from 18-month-old WT and Cd $37^{-1-}$ mice, as detected by Western blot ( $n \geq 4$ mice per genotype). p-STAT3 protein levels were normalized to total STAT3 levels. Data represent mean \pm SEM. ${ }^{*} P<0.05$, Mann-Whitney nonparametric test.

Constitutive activation of the IL-6 signaling pathway in Cd37-1lymphomas. To gain insight in the mechanism underlying the spontaneous lymphoma development in $\mathrm{Cd} 37^{-/}$mice, we performed quantitative PCR using a Mouse Oncogenes \& Tumor Suppressor Genes PCR Array on mLNs from tumor-bearing $C d 37^{-/}$mice and age-matched WT littermates. Among the 84 cancer-associated genes, 6 genes were upregulated approximately 2 -fold in the neoplastic $C d 37^{-/-}$tissue compared with that in normal WT mLN tissue (Supplemental Table 2). Three of the upregulated genes were prominent members of the IL-6 signaling pathway (Jun, Akt1, Stat3; Figure 4A and Supplemental Table 2). We could verify this at the mRNA level (data not shown) and protein level by tissue immunohistochemistry analyses demonstrating increased STAT3 and AKT phosphorylation (indicative of STAT3 and AKT activation) in lymphoma tissues of $C d 37^{-/}$mice (Figure 4B). Furthermore, levels of phosphorylated STAT3 (p-STAT3) were enhanced in Cd37-lymphoma cells (derived from mLNs or spleens) compared with those in WT controls, as determined by quantitative Western blot analysis, indicating that the IL- 6 signaling pathway is activated in Cd37/- lymphoma cells (Figure 4, C and D). Expression of the protooncogene Ctnnb1, encoding $\beta$-catenin (35), was also enhanced, whereas expression of the proapoptotic gene Casp 8 , encoding cas- pase-8 (36), was decreased in Cd37/- tumors (Supplemental Figure 6). The role of IL-6 in lymphomagenesis was further validated by quantifying IL-6 levels in sera of $C d 37^{-}$and WT mice during aging. $C d 37^{-/}$mice with lymphomas exhibited increased levels of IL-6 in sera compared with mice without tumors (Figure 5A). In addition, tumor cells derived from $\mathrm{Cd}_{37^{-/}}$mice spontaneously produced IL-6 ex vivo in contrast to $\mathrm{mLN}$-derived cells from WT mice (Figure 5B), which was confirmed by immunohistochemistry studies (Figure 5C). To verify whether IL-6 was involved in the enhanced phosphorylation of both STAT3 and AKT, blocking IL-6 monoclonal antibody or isotype control monoclonal antibody was added to freshly isolated Cd37- tumor cells. Blocking of IL-6 reduced STAT3 and AKT phosphorylation, as assessed by intracellular flow cytometry (Figure 5D). These reduced levels of p-STAT3 and phosphorylated AKT (p-AKT) after blocking IL-6 were associated with decreased viability of the neoplastic cells during ex vivo cell culture (Figure 5E). Together, these data support the importance of IL-6 in B cell lymphoma development in Cd37/- mice.

$C D 37$ regulates $I L-6$ responses through direct interaction with SOCS3. Since IL-6 signaling appeared to be directly correlated with B cell lymphomagenesis in $C d 37^{-/}$mice, we first evaluated whether the components of the IL-6R complex were differentially 
A
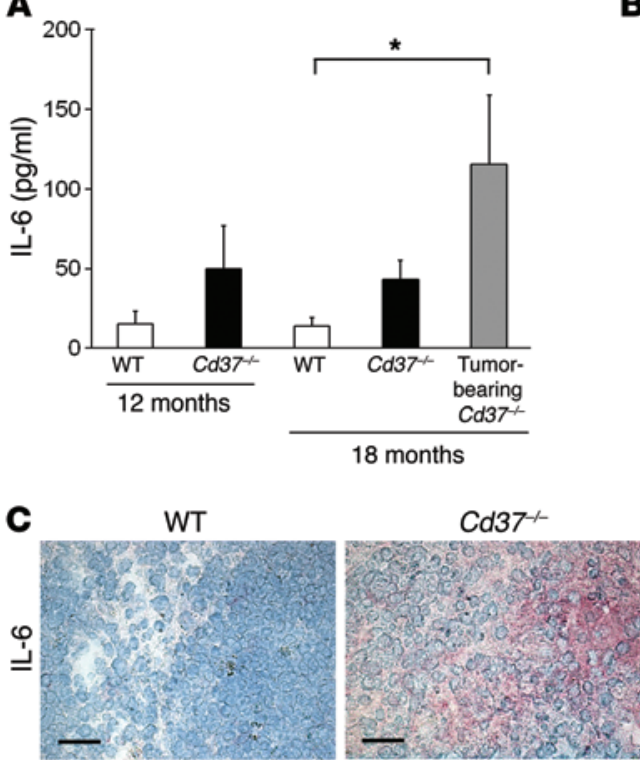

E

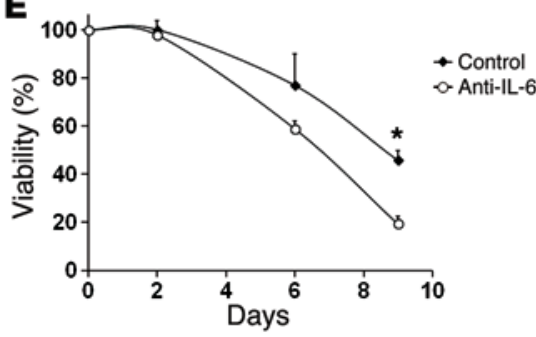

B

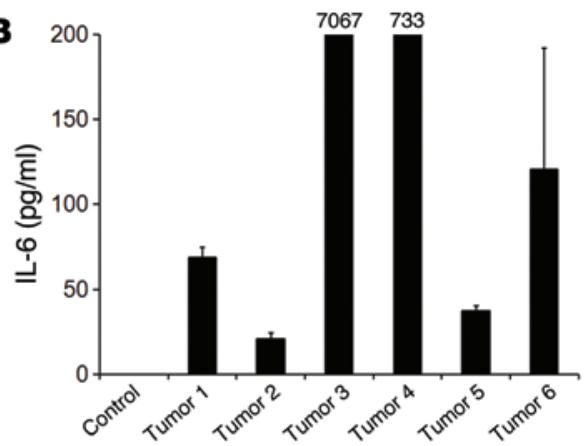

D

WT
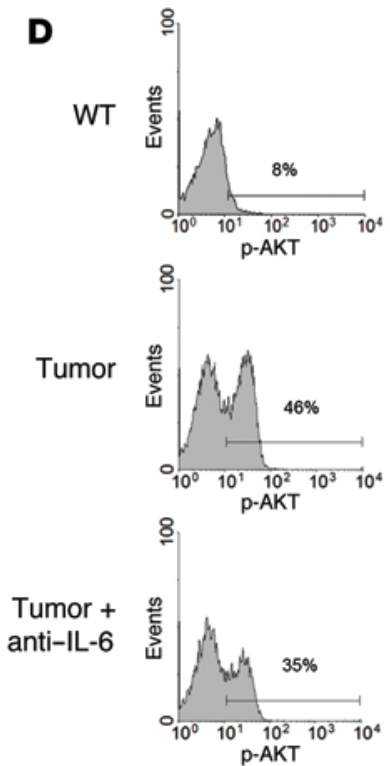
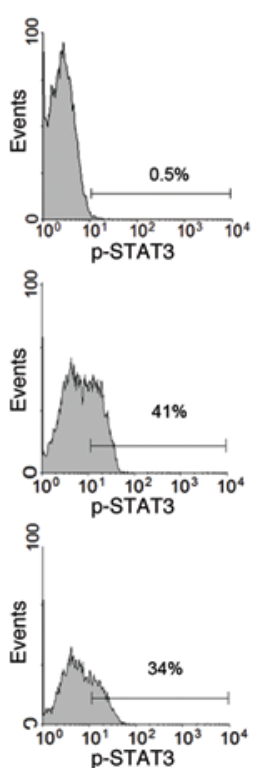

Figure 5. Blocking IL-6 supports B cell lymphoma cell viability. (A) Lymphoma-bearing $C d 37^{-/-}$mice contain significantly $\left({ }^{*} P=0.016\right.$, Kruskal-Wallis 1-way ANOVA test) higher IL-6 levels than aged-matched WT mice ( $n=6$ mice per strain aged 12 months, $n=16$ mice per strain aged 18 months). Data represent mean \pm SEM. (B) IL-6 produced by ex vivo-cultured lymphoma cells from 6 individual $\mathrm{Cd} 37^{-1-}$ mice versus WT controls $(n=3)$. Data represent mean \pm SD. (C) Immunohistochemistry analysis of IL- 6 in $\mathrm{mLN}$ tissues from 18-month-old WT and Cd37-/- mice $(n=6)$. Scale bar: $50 \mu \mathrm{m}$. (D) B cells of $\mathrm{mLNs}$ from 18-month-old WT and $\left[\mathrm{d}_{3} 7^{-/-}\right.$mice were stained for intracellular p-AKT and p-STAT3 and analyzed by flow cytometry. The percentage of $\mathrm{p}-\mathrm{AKT}^{+}$and $\mathrm{p}-\mathrm{STAT3}^{+}$ lymphoma cells is reduced by IL-6neutralizing antibodies (anti-IL-6) (bottom). (E) Viability of lymphoma cells of $C d 37^{-1-}$ mice upon ex vivo culture with anti-IL-6, as detected by trypan blue exclusion at different time points. Data represent mean $\pm \mathrm{SEM}$. ${ }^{*} P<0.0001$. Experiments were performed 3 times with at least 3 mice per genotype. Unpaired Student's $t$ test was used for statistical analysis. expressed on B cells in the absence of CD37. We found no differences in expression of IL-6R $\alpha$ or its signaling chain gp130 between $\mathrm{B}$ cells derived from aged $\mathrm{Cd} 37^{-/-}$mice and WT mice (Figure 6A). These data indicate that the IL-6R itself is functional but that it is likely that the negative feedback machinery of the IL-6 pathway is disturbed in $\mathrm{B}$ cells from $\mathrm{Cd} 37^{-/-}$mice. To address the possibility that $C d 37^{-/-}$B cells have evolved to escape control of SOCS3, the major negative feedback regulator of IL- 6 signaling, we determined the expression levels of SOCS3 in B cells from $\mathrm{Cd} 37^{-/}$mice. Surprisingly, Cd37/- lymphoma cells from 18-month-old $C d 37^{-1-}$ mice contained very high basal levels of SOCS3 in contrast to those from WT controls (Figure 6B). Based on the finding that this high SOCS3 expression in $C d 37^{-/}$lymphomas was unable to inhibit the IL-6 signaling pathway (Figure 5), we hypothesized that CD37 itself might interfere with this negative feedback machinery. This was investigated by coimmunoprecipitation experiments, which revealed that $\mathrm{CD} 37$ was indeed able to directly interact with SOCS3 (Figure 6C). Next, we studied the localization of endogenous CD37 and SOCS3 in human B cells using confocal microscopy. In the absence of IL-6, SOCS3 was predominantly localized in the cytoplasm (Figure 6D). IL-6 stimulation clearly induced translocation of SOCS3 to CD37 microdomains in the plasma membrane of human B cells, and significantly more translocation was observed in IL-6-stimulated B cells than in unstimulated
B cells (Figure 6D). Clear colocalization was observed between CD37 and IL-6R $\alpha$ in the plasma membranes of resting human B cells (Manders coefficient: 0.69; data not shown), confirming that these microdomains were also enriched in the IL-6R complex (Figure 6E). Human pre-B cells, which have very low levels of endogenous CD37 but do express the main components involved in IL-6R signaling (Supplemental Figure 7), were transfected with CD37-GFP and analyzed for activation of the IL-6 pathway. We observed CD37-expressing B cells to contain lower levels of p-STAT3 upon IL-6 stimulation than CD37- B cells (Figure 6F), thus (partially) rescuing the phenotype of $C d 37^{-/}$cells. Together, these data support the role for CD37 in inhibition of the IL-6 signaling pathway through its interaction with SOCS3.

To prove that the development of $\mathrm{B}$ cell malignancies in $C d 37^{-/-}$mice was dependent on enhanced IL-6 signaling in vivo, we generated $C d 37 \times$ Il 6 double-knockout $\left(C d 37^{--} x I l 6^{--}\right)$mice and investigated whether ablation of CD37 in the absence of IL-6 could prevent the development of spontaneous malignancies during aging. Strikingly, $\mathrm{Cd} 37^{-/}-\mathrm{xIl} 6^{-/-}$mice were rescued from cancer development (only 1 of 26 [4\%] of mice developed lymphoma; Figure 6G and Supplemental Table 1), confirming that $C d 37^{-/}$mice develop B cell lymphomas in an IL-6-dependent manner.

Loss of CD37 correlates with upregulated IL-6 signaling and poor survival in patients with DLBCL. To validate the role of CD37 in 
A
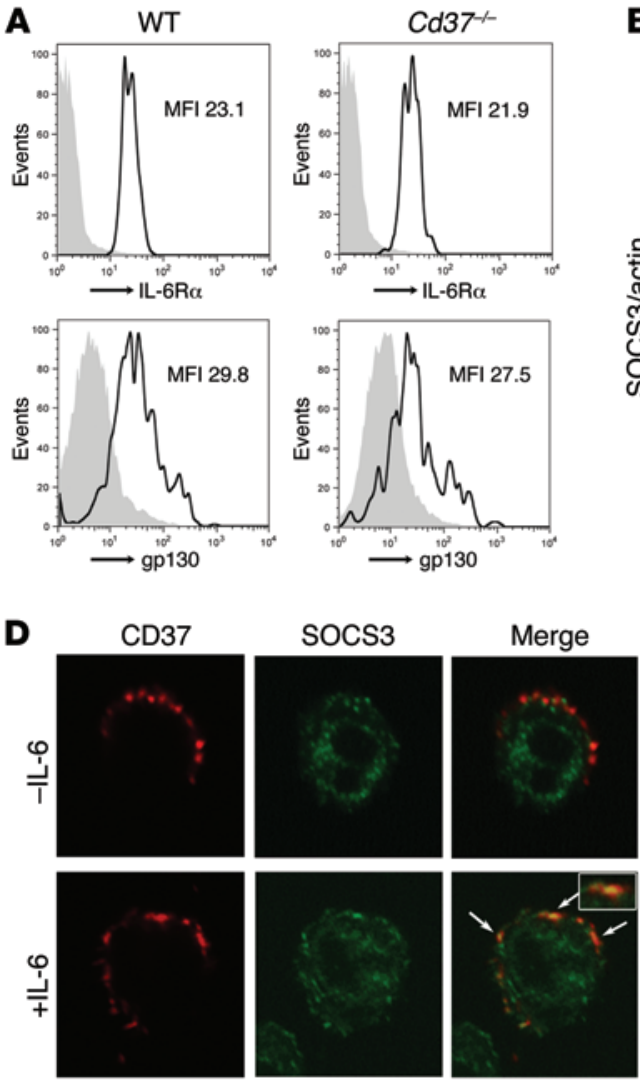

E

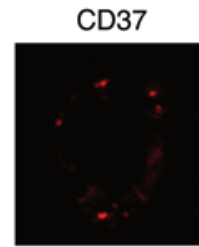

CD37

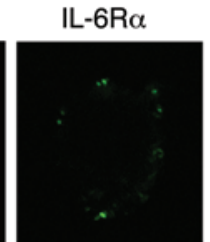

Control
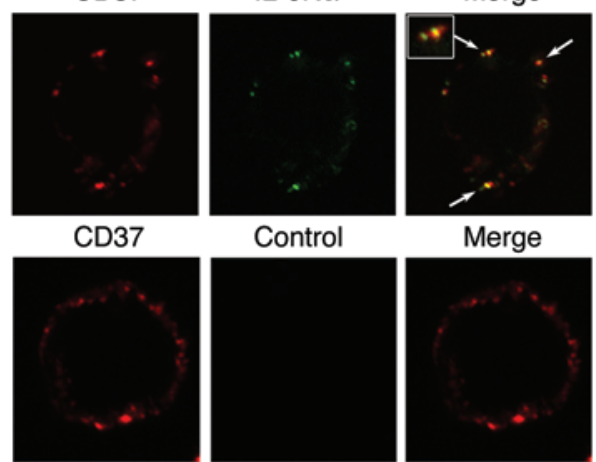

Merge

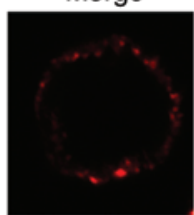

B
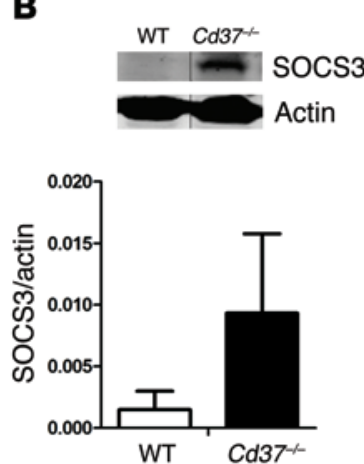

C

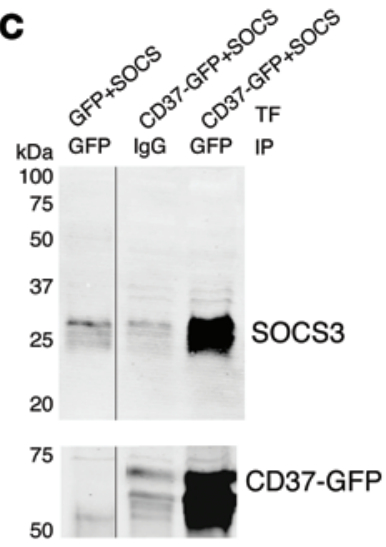

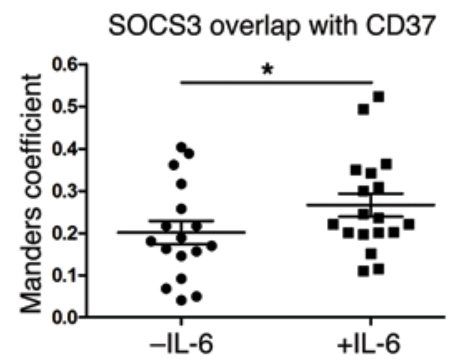

$\mathbf{F}$
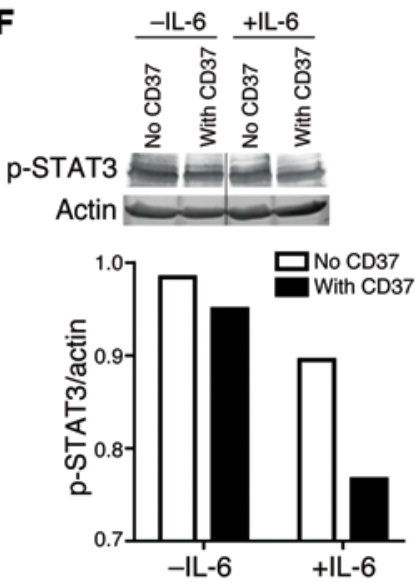

G

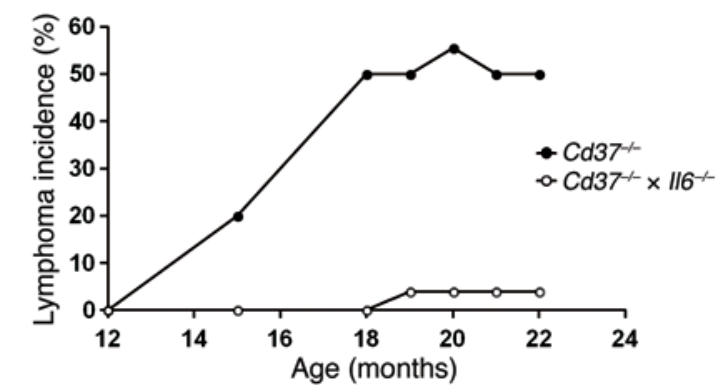

Figure 6. CD37 controls the IL-6 pathway via SOCS3, and lymphoma development is dependent on IL-6 in vivo. (A) Comparable surface expression of IL-6R $\alpha$ and gp130 in the plasma membrane of B220 IgA+ B cells from 18-month-old WT and Cd37-- mice analyzed by flow cytometry (specific antibody staining [black] versus isotype control antibody [gray]). MFI, mean fluorescence intensity. (B) Protein levels of SOCS3 in mLN cells from 18-month-old WT and Cd37-/- mice, as detected by Western blot (noncontiguous lanes from the same gel). SOCS3 protein levels were normalized to actin levels ( $n=5$ mice per genotype). Data represent mean \pm SEM. (C) HEK293 cells were cotransfected (TF) with CD37-GFP (or GFP alone) and SOCS3, followed by immunoprecipitation with anti-GFP or isotype control IgG. Western blots were probed with anti-SOCS3 (26 kDa) and anti-GFP (CD37-GFP, 60-75 kDa, due to heavy glycosylation, ref. 66; noncontiguous lanes from the same gel). (D) Colocalization of CD37 (red) and SOCS3 (green) or control antibody was studied on human JY B cells without (-) or with (+) IL-6 stimulation by confocal microscopy. Colocalization is shown in yellow (white arrows). Manders coefficient was determined with $n \geq 17$ cells from 3 independent experiments (each dot represents a single cell). Data represent mean $\pm \mathrm{SEM} .{ }^{*} P<0.05$, Mann-Whitney nonparametric test. (E) Colocalization of CD37 (red) and IL-6R $\alpha$ (green) or control antibody was studied on human JY B cells by confocal microscopy. Colocalization (yellow) was independent of IL-6 stimulation. Data are shown for B cells without IL-6 stimulation. (F) p-STAT3 (76-78 kDa) levels in NALM-6 cells, which were mock transfected or transfected with CD37-GFP, with or without IL-6 stimulation for 60 minutes. Actin ( $42 \mathrm{kDa}$ ) was used as loading control (noncontiguous lanes from the same gel). (C) $C d 37^{-1-} \times 1 / 6^{-1-}$ mice are protected against development of B cell lymphoma $(n=26)$ (see Supplemental Table 1 for details). Original magnification: $\times 630(\mathbf{D}$ and $\mathbf{E}) ; \times 1,260$ (insets, $\mathbf{D}$ and E).

human B cell lymphoma, we investigated CD37 surface expression in biopsies of 47 patients with de novo GC B cell-like DLBCL (GCB-DLBCL) and 50 patients with activated B cell-like DLBCL (ABC-DLBCL) uniformly treated with standard R-CHOP chemo- therapy. Cell-of-origin classification of GCB-DLBCL was determined by gene expression profiling in these patients (37). Approximately $32 \%$ of GCB-DLBCL tumors and $46 \%$ of ABC-DLBCL tumors expressed ample CD37 protein at the cell membrane, 
A
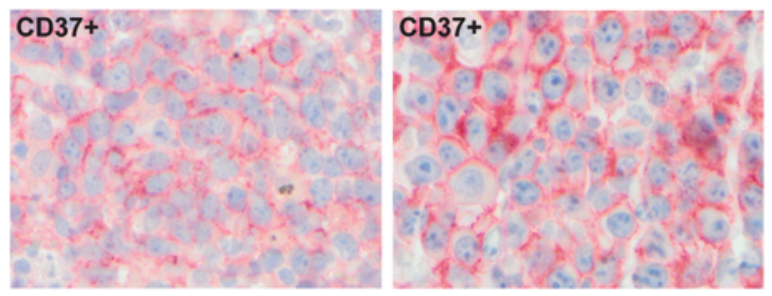

CD37-

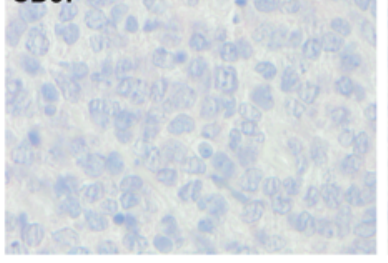

CD37-

B

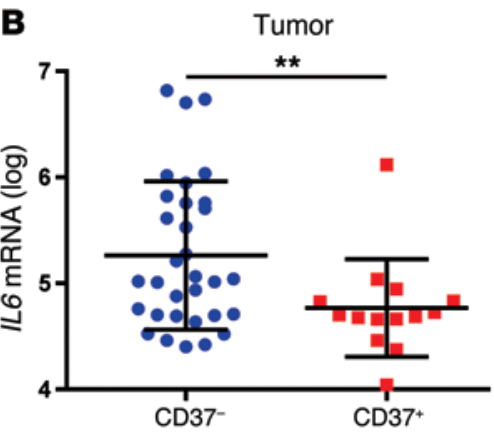

D

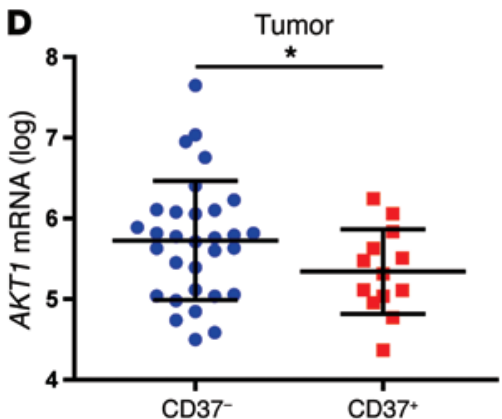

C

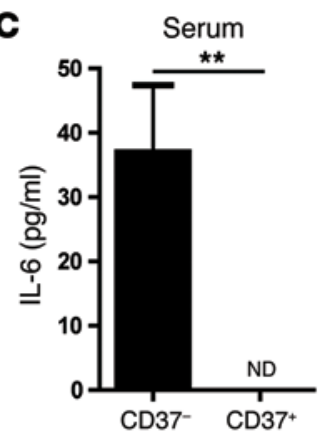

E

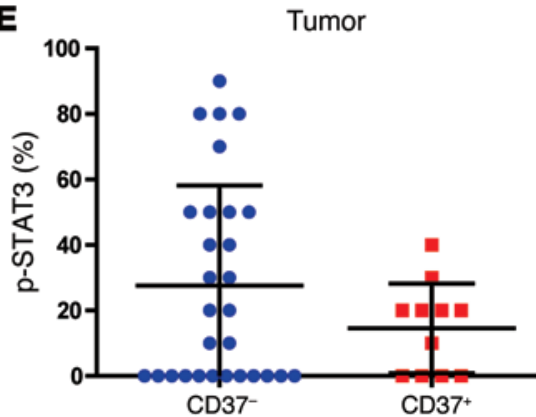

Figure 7. Loss of CD37 in lymphomas of patients with DLBCL correlates with upregulated IL-6 signaling.

(A) CD37 immunohistochemistry staining (red) of GCB-DLBCL biopsies. Cell nuclei were counterstained with hematoxylin (blue). Representative stainings of $2 \mathrm{CD}^{-} 7^{+}$and $\mathrm{CD} 37^{-}$tumors are shown. Original magnification: $\times 100$. (B) Expression of IL6 mRNA levels in lymphomas of 47 patients with GCB-DLBCL expressing (CD37 ${ }^{+}, n=15$; red dots) or not expressing (CD37 $-n=32$; blue dots) cell surface CD37. Data represent mean $\pm \mathrm{SD} .{ }^{*} P=0.0098$; unpaired Student's $t$ test. (C) Expression of IL-6 protein levels in sera of patients with DLBCL with CD37- tumors $(n=3)$. Cytokine levels of patients with DLBCL with $\mathrm{CD} 7^{+}$tumors were not detectable above background signal (ND) $(n=5)$. Data represent mean \pm SEM. ${ }^{*} P=0.0084$, Mann-Whitney nonparametric test was used for statistical analysis. (D) Expression of AKT1 mRNA in lymphomas of patients with GCB-DLBCL without CD37 expression (CD37- $n=32$, blue dots) compared with $\mathrm{CD}^{+} 7^{+}$tumors ( $n=15$, red dots). Data represent mean $\pm S D$. ${ }^{*} P=0.0476$, unpaired Student's $t$ test. (E) Nuclear expression of (activated) p-STAT3 protein in lymphomas of patients with GCB-DLBCL with CD37- tumors ( $n=32$, blue dots) compared with $\mathrm{CD} 37^{+}$tumors ( $n=15$, red dots). Data represent mean $\pm \mathrm{SD} . P=0.0915$, unpaired Student's $t$ test. whereas up to $68 \%$ of GCB-DLBCL tumors and $54 \%$ of ABCDLBCL tumors totally lost CD37 protein expression (2 representative $\mathrm{CD} 7^{+}$and CD37- GCB-DLBCL tissues are shown in Figure 7A).

Consistent with $\mathrm{B}$ cell lymphoma development in $\mathrm{Cd} 37^{-/-}$mice, loss of CD37 expression in human B cell lymphomas correlated with upregulated IL6 mRNA levels in the tumor $(P=0.0098$; Figure $7 \mathrm{~B})$ and with presence of IL- 6 protein in sera of those patients $(P=0.0084$; Figure 7C). Moreover, CD37- DLBCL tumors had significantly higher $A K T 1 \mathrm{mRNA}$ levels $(P=0.0476$; Figure $7 \mathrm{D})$ and a trend toward higher mean value of nuclear expression of p-STAT3 $\left(P=0.0915\right.$; Figure 7E) compared with $\mathrm{CD} 37^{+}$tumors.

Strikingly, Kaplan-Meier analysis showed a significant worse overall survival (OS; hazard ratio, 3.14; 95\% CI, 1.17-8.41; $P=0.02$ ) and progression-free survival (PFS; hazard ratio, 2.58; 95\% CI, 0.99-6.68; $P=0.05$ ) in patients with GCB-DLBCL with CD37tumors compared with patients with GCB-DLBCL with $\mathrm{CD} 37^{+}$ tumors (Figure 8, A and B). The median OS was 86 months for patients with $\mathrm{CD} 37^{-}$tumors, and this median OS was not reached for patients with $\mathrm{CD} 7^{+}$tumors, and the median PFS was 63.8 months for patients with CD37 tumors, and this median OS was not reached for patients with $\mathrm{CD} 37^{+}$tumors. These findings were confirmed in patients with ABC-DLBCL. Patients with ABCDLBCL with CD37 ${ }^{-}$tumors had a worse OS (hazard ratio, 2.87; 95\% CI, 1.13-7.29; $P=0.03$ ) and PFS (hazard ratio, 3.71; 95\% CI, $1.55-8.89 ; P=0.003)$ compared with patients with $\mathrm{CD} 37^{+}$tumors (Figure 8, C and D). In summary, loss of CD37 in tumors of both patients with GCB-DLBCL and patients with ABC-DLBCL is significantly correlated with activation of the IL- 6 signaling pathway and decreased survival.

\section{Discussion}

IL-6 has emerged as a critical tumor-promoting cytokine in different types of human cancer, including B cell malignancies $(8,38)$. Although IL- 6 and its downstream effectors are widely studied as therapeutic targets for patients with B cell malignancies, very little is known about the underlying mechanisms that induce constitutive IL-6 signaling in these tumor cells. Here, we demonstrate that deficiency of CD37 leads to spontaneous development of GC- 
A

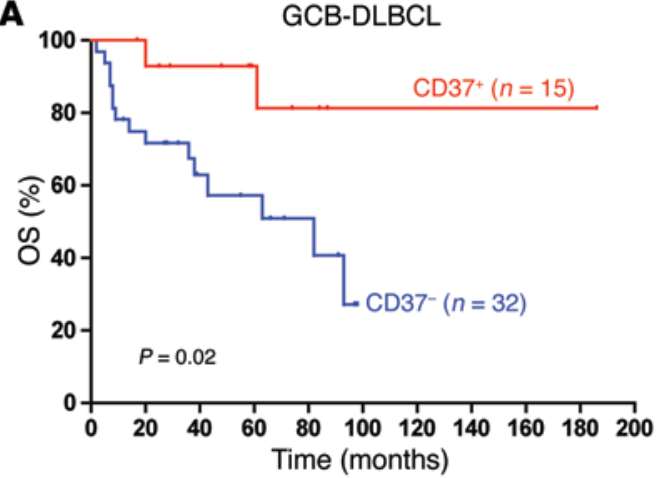

C

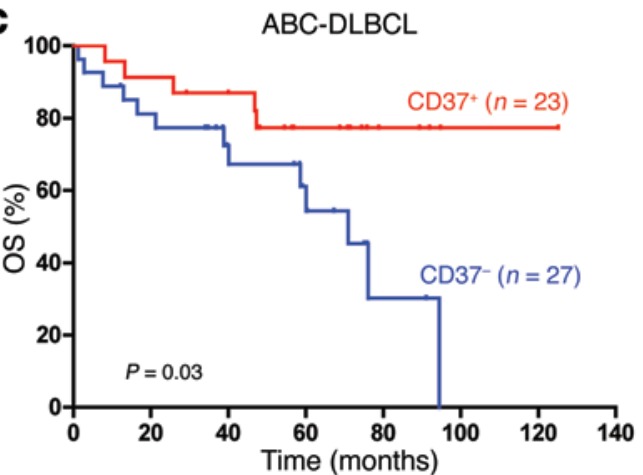

B

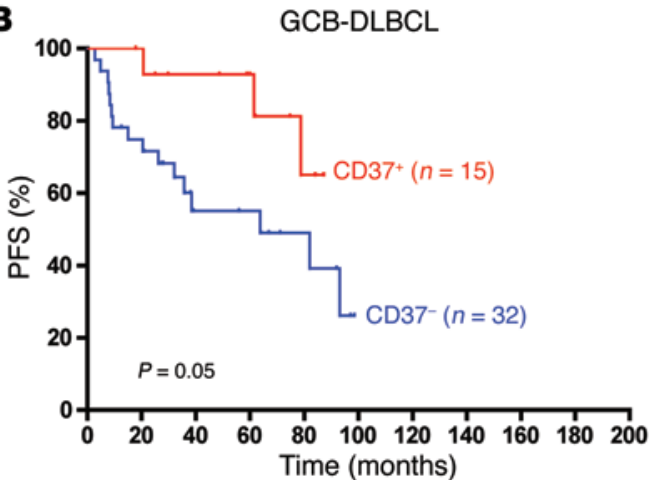

D

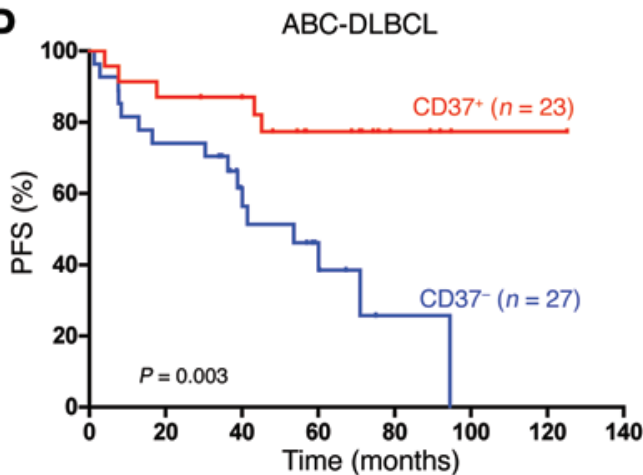

Figure 8. Loss of CD37 in lymphomas of patients with DLBCL correlates with worse survival. (A and B) Loss of CD37 correlates with significantly poor (A) OS $(P=0.02)$ and (B) PFS $(P=0.05)$ in 47 patients with GCB-DLBCL. (C and $\mathbf{D})$ Loss of CD37 correlates with significantly poor (C) OS $(P=0.03)$ and (D) PFS $(P=0.003)$ in 50 patients with $A B C-D L B C L$. Log-rank (Mantel-Cox) test was used for statistical analysis. derived B cell lymphoma in an IL-6-dependent manner. CD37 is a regulator of SOCS3 and thereby interferes with activation of the IL-6 signaling pathway in B cells.

Gene expression profiling of tumor suppressors and oncogenes in $C d 37^{-/}$lymphomas revealed upregulation of Akt1, Stat3, and Jun, which play a central role in the IL-6 signaling pathway and have been implicated in cancer cell survival $(18,19)$. The constitutive activation of AKT in $C d 37^{-/-}$lymphomas corresponds to previous work showing that CD37 regulates AKT signaling, leading to B cell survival $(27,39)$. Importantly, in $68 \%$ of human GCB-DLBCL tumors and in 54\% of human ABC-DLBCL tumors, CD37 expression was lost, which correlated with increased IL-6 levels and elevated levels of AKT1 mRNA and p-STAT3, validating the findings in $C d 37^{-/-}$mice.

$B c l 2$ transgenic mice develop lymphoma at low frequencies (15\%-20\%) (40) compared with the 50\% incidence observed in the $C d 37^{-1-}$ mouse strain at the same age and background. We did not detect enhanced $\mathrm{Bcl} 2$ expression in $\mathrm{Cd} 37^{-/}$lymphomas (Supplemental Table 2), indicating that the tumor suppressor activity of CD37 is independent of BCL-2. B cells from young $C d 37^{-/-}$mice are prone to differentiate toward IgA-producing plasma cells, due to the high IL-6 levels that circulate in vivo (28). Although constitutive expression of the BCL-2 protein has been reported in many human FL and DLBCL tumors (41), we now provide a CD37dependent mechanism for B cell lymphomagenesis. Our findings are in line with those in studies showing that Il6 transgenic mice are prone to develop FL, plasmacytoma, and DLBCL (42) and IL-6 signaling in B cell malignancies can be independent of BCL-2 (43). The $C d 37^{-1-}$ lymphoma model may serve as a new model for human B cell lymphomas in which the IL-6 signaling pathway is constitutively activated. It will be interesting to investigate other
IL-6 family members in $C d 37^{-/-}$mice, including IL-11, which was recently reported to play a major role in inflammation-induced tumors due to constitutive STAT3 activation (44).

Importantly, we uncovered the underlying molecular mechanism of lymphoma formation in $\mathrm{Cd} 37^{-/-}$mice by investigating SOCS3, the major negative regulator of the IL- 6 signaling pathway (45). SOCS3 is a tumor suppressor, as demonstrated in conditional Socs3-deficient mice (Socs3 deletion in gastrointestinal epithelial cells) that develop gastric cancer (46). In human B cell malignancies, hypermethylation of SOCS3 leading to hyperactivation of STAT3 has been correlated with worse patient outcome $(47,48)$. In contrast, increased SOCS3 expression has been reported in melanoma (49) and breast cancer (50). Furthermore, increased SOCS3 mRNA levels in peripheral blood of patients with nonHodgkin lymphoma have been found to correlate with worse disease outcome (51). We found elevated levels of SOCS3 in Cd37-/tumors compared with those in WT control tissues, indicating that negative feedback regulation by SOCS3 is defective in the absence of CD37. Indeed, we observed that CD37 directly interacts with SOCS3. Based on these observations, we propose the following model for the role of CD37 in the IL-6 signaling pathway (Supplemental Figure 8). Upon activation of the IL-6R complex, AKT and STAT3 are phosphorylated, leading to production of SOCS3 and subsequent inhibition of the IL- 6 signaling cascade by SOCS3 binding to gp130. CD37 facilitates this inhibition by binding SOCS3 and stabilizing the IL-6R complex in the plasma membrane. In $C d 37^{-/-}$B cells, SOCS3 cannot bind efficiently to the IL-6R complex, resulting in defective feedback inhibition. Consequently, SOCS3 will accumulate in the cytoplasm due to continuous activation of the IL- 6 signaling pathway through autocrine IL- 6 production by the tumor cells. In conclusion, the IL- 6 
signaling pathway is constitutively activated, which is caused by defective regulation by SOCS3 due to CD37 deficiency, resulting in malignant B cell transformation and tumor cell survival.

The association of tetraspanins with tumor progression has been established, although the underlying mechanisms have not been fully resolved $(23,24,52)$. Little is known about tetraspanin involvement in human B cell lymphomagenesis. CD81 protein is expressed at high levels in normal GC B cells and in DLBCL (53), whereas downregulation of CD9 has been related to lymphoma progression (54). Our data now demonstrate that loss of CD37 in DLBCL is directly correlated with worse OS and PFS, which shows that CD37 may serve as a novel prognostic marker for patients with DLBCL. Sequencing of the CD37 gene in human B cell lymphomas did not reveal obvious mutations (data not shown). Thus, we postulate that CD37 deficiency in human DLBCL is most likely caused by involvement of microRNAs or epigenetic changes, which have been reported for different tumor suppressor genes as an alternative mechanism of gene silencing in cancer $(55,56)$. To the best of our knowledge this study is the first to demonstrate that tetraspanin deficiency itself can lead to tumor development. In most human B cell malignancies, including chronic lymphocytic leukemia and different types of non-Hodgkin lymphoma, CD37 is highly expressed by tumor cells (57). Since CD37 can directly couple to B cell survival, CD37 targeting is currently under investigation in clinical trials in patients with B cell malignancies $(25,58,59)$. Our study now demonstrates that expression of CD37 is completely lost in 68\% of GCB-DLBCL tumors and in $54 \%$ of ABC-DLBCL tumors, which has important consequences for selecting patients for CD37-based immunotherapies. Furthermore, we anticipate that patients with CD37- lymphomas may particularly benefit from therapies inhibiting the IL-6 pathway, including monoclonal antibodies that target IL-6 and/or IL-6R (8, 19) and JAK1/2 inhibitors that can reduce p-STAT3 and IL- 6 levels in patients with myeloproliferative disorders (60).

Taken together, this study demonstrates that tetraspanin CD37 protects against development of GC-derived B cell lymphoma. We found that CD37 interacts with SOCS3 and thereby induces feedback inhibition of the IL-6 signaling pathways via STAT3 and AKT, providing direct mechanistic insight into the regulation of the IL-6 signaling pathway in B cell biology and lymphomagenesis in vivo. This study is of major clinical relevance in human B cell lymphoma, since CD37 expression in patients with DLBCL is directly correlated with OS and PFS. Moreover, the discovery that $>50 \%$ of DLBCL tumors are CD37- has important consequences for CD37directed immunotherapies that are currently in clinical trials and provides a strong rationale for blocking the IL- 6 pathway in patients with $\mathrm{CD}^{-} 7^{-} \mathrm{B}$ cell malignancies as therapeutic intervention.

\section{Methods}

Mice. $C d 37^{-/}$mice were generated by homologous recombination (32) and backcrossed to the C57BL/6J background (29). $\mathrm{Cd} 37^{-1-} x \mathrm{Il} 6^{-/-}$mice were generated by crossing $C d 37^{--}$mice with $\mathrm{Il6}^{--}$mice (61). WT littermates were matched for age and gender. BALB/cAnNRj-Foxn $1^{n u}$ (nude) mice were purchased from Janvier Labs, and NOD/SCID/IL2 R $\gamma$ null (NSG) mice were originally purchased from The Jackson Laboratory. $\mathrm{Cd} 37^{+/+}, \mathrm{Cd} 37^{-/-}, \mathrm{Cd} 37^{-/} \times \mathrm{Il} 6^{-/-}$, and NSG mice were bred in the Radboud University Medical Center Central Animal Laboratory. All mice were housed in top-filter cages and fed a standard diet with freely available water and food.

Patients. Biopsies of 47 patients with de novo GCB-DLBCL and 50 patients with $A B C-D L B C L$ were obtained and stained for CD37 using immunohistochemistry. Patients were diagnosed between 2001 and 2008, lymphomas were classified according to the World Health Organization 2008 classification criteria, and the pathology was reviewed and validated. The median age was 61 years, and all patients underwent standard R-CHOP chemotherapy. Thirty-five patients had died by the last follow-up. The median follow-up interval for the 62 censored patients was 59.6 months (range from 12.2 to 186.7 months). Some of the patients with GCB-DLBCL and ABC-DLBCL had serum available, which was used to evaluate IL-6 levels.

Histology and immunohistochemistry. Collected tissues were fixed in phosphate-buffered $4 \%$ paraformaldehyde (PFA) and embedded in paraffin wax. Serial $5-\mu \mathrm{m}$ tissue sections were stained with $\mathrm{H} \& \mathrm{E}$ prior to microscopical analysis. Two independent pathologists (M.J. Gijbels and K.M. Hebeda) classified the lymphomas as described previously (34). Immunohistochemical stainings were performed on OCT-embedded frozen tissue sections. Tissue slides were dried at room temperature for 30 minutes, fixed in $2 \%$ PFA for 10 minutes, and treated with $3 \% \mathrm{H}_{2} \mathrm{O}_{2}$ in methanol for 15 minutes at room temperature. After blocking Fc $\gamma$ receptors with normal serum in which the secondary antibody was produced, tissue sections were stained against IL-6 (MP5-32C11, BD Pharmingen), B220 (RA3-6B2, BD Pharmingen), CD19 (1D3, eBioScience), CD3 (Cd3-12, Serotec), F4/80 (CI:A3-1, Serotec), CD68 (FA-11, Serotec), Gr1 (RB6-8C5, BD Pharmingen), PNA-biotin (Sigma-Aldrich), GL7 (GL-7, eBioScience), CD138 (281-2, Biolegend), IgA (C10-1, BD Pharmingen), phospho-STAT3 (Tyr ${ }^{705}$, D3A7, Cell Signaling Technology), and phospho-AKT (Ser ${ }^{473}$, D9E, Cell Signaling Technology) for 1 hour at room temperature. Subsequently, tissue slides were subjected to incubation with appropriate biotinylated secondary antibodies for 45 minutes at room temperature, followed by avidin-biotin complex detection (Vectastain, Vector), according to the manufacturer's instructions. Complexes were visualized using Fast Red Substrate, counterstained with hematoxylin, and mounted with Kaiser's Glycerin Gelatin (Merck). Immunohistochemistry staining on human lymphoma tissues of patients with DLBCL was performed on formalin-fixed and paraffin-embedded tissue microarrays following the manufacturer's instructions using monoclonal antibody specific for CD37 (clone 2B8, ThermoFisher Scientific) and p-STAT3 (62), followed by hematoxylin counterstaining. Assessment of CD37 and p-STAT3 protein expression was performed by two pathologists (K.J. Jabbar and K.H. Young) individually blinded from the clinical outcomes. The interobserver agreement was $98 \%$ for the samples, and disagreements were resolved by joint review on a multihead microscope.

Immunofluorescent staining of immunoglobulins. Cryostat 4- $\mu \mathrm{m}$ sections were dried at room temperature for 30 minutes and fixed in acetone for 10 minutes at $-20^{\circ} \mathrm{C}$. Subsequently, tissue sections were treated with $5 \%$ normal horse serum for 30 minutes at room temperature. After blocking, tissue sections were stained with AffiniPureF $\left(a b^{\prime}\right)_{2}$ fragment goat anti-mouse IgG $(2 \mu \mathrm{g} / \mathrm{ml}$, Jackson ImmunoResearch Laboratories), goat anti-mouse IgM ( $5 \mu \mathrm{g} / \mathrm{ml}$, Vector Laboratories), or rat anti-mouse IgA (C10-1, $2 \mu \mathrm{g} / \mathrm{ml}$, BD Pharmingen) for 45 minutes at room temperature, followed by incubation with appropriate secondary antibodies (Alexa Fluor 488, Life Technologies) for 45 minutes at room 
temperature. Sections were fixed in 1\% PFA, enclosed in Mowiol (EMD Biosciences), and imaged by fluorescent microscopy (Leica DM).

Flow cytometry and antibodies. Single-cell suspensions were stained for 30 minutes at $4^{\circ} \mathrm{C}$ in PBS, $1 \%$ BSA, and $2 \%$ normal goat serum, with the following primary mouse antibodies: anti-CD19-APC (1D3, eBioscience), anti-CD3-APC (145-2C11, BD Pharmingen), anti-B220-FITC (RA3-6B2, Biolegend), anti-IgA-FITC (mA-6E1, eBioscience), IgDFITC (11-26c.2a, BD Pharmingen), anti-CD86-PE (GL1, BD Pharmingen), anti-MHC-II-PE (M5/114.15.2, eBioscience), anti-CD40-PE (3/23, BD Pharmingen), anti-CD43-PE (S7, BD Pharmingen), antiB220-Alexa Fluor 647 (RA3-6B2, BD Pharmingen), biotinylated antiIgM (Vector Laboratories), anti-IgA (C10-1, BD Pharmingen), PNA (Sigma-Aldrich), anti-CD138 (281-2, BD Pharmingen), anti-CD37 (WR17, made in-house), anti-IL-6R $\alpha / C D 126$ (D7715A7, Biolegend), or anti-gp130 (M-20, Santa Cruz Biotechnology). This was followed by incubation with streptavidin conjugated to PE or PerCP or appropriate secondary antibodies (PerCP, BD Pharmingen, or anti-rabbit IgG-Alexa Fluor 488, anti-mouse IgG2a-Alexa Fluor 647, anti-mouse IgG1-Alexa Fluor 488, Life Technologies). Intracellular stainings for p-STAT3 $\left(\mathrm{Tyr}^{705}\right)$ and p-AKT $\left(\mathrm{Ser}^{473}\right)$ were performed as described previously (27). Stained cells were analyzed using a FACSCalibur (Becton Dickinson) and FlowJo software version 9.6 (TreeStar Inc.).

Assessment of immunoglobulin heavy chain rearrangements. IGH rearrangements were assessed using the standardized multiplex PCR protocols (35 PCR cycles) developed by the BIOMED-2 concerted action BMH4-CT98-3936 (63). The IGH complete (VDJ) rearrangements were assessed in the 3 framework regions in the IGH variable genes. Multiplex PCR products were monitored by GeneScan analysis on an ABI 3730 platform (Life Technologies) and processed by the Genemapper software (version 4.0, ABI prism). Interpretation of the clonality findings was performed according to the EuroClonality guidelines (64). This test to perform clonality assessment on human samples is validated for use in murine samples.

Adoptive transfer experiments. Tumors from $\mathrm{Cd} 37^{-/}$mice were incubated with collagenase and DNAse in order to obtain single-cell suspensions. Freshly isolated tumor cells $\left(5 \times 10^{6}\right)$ were injected subcutaneously into the flanks or intravenously into the tail veins of gender-matched 6- to 8-week-old $C d 37^{-/}$, WT, or immunocompromised recipients. Tumor growth was measured over time. In case of subcutaneous injection, human endpoint was reached at tumor size $>2 \mathrm{~cm}^{2}$.

RNA isolation and quantitative PCR analysis. For PCR-based gene profiling, the following protocol was used. Total RNA was prepared from $C d 37^{-/-}$tumor or WT control mLNs using the MagNA Lyser Instrument (Roche) and the RNeasy Kit (Qiagen). Total RNA was reverse transcribed to cDNA using the RT2 First-Strand Kit (Qiagen) following the manufacturer's instructions. Array plates of Mouse Oncogenes \& Tumor Suppressor Genes PCR Array (SA Bioscience) were run according to the manufacturer's instructions using the ABI/Prism 7000 Sequence Detection System (Applied Biosystems). The $\Delta \mathrm{Ct}$ value was calculated by determining the difference between the $\mathrm{Ct}$ value of the gene of interest and the average Ct value of 5 different housekeeping genes (Gusb, Hprt1, Hsp90ab1, Gapdh, and Actb). The $\Delta \Delta \mathrm{Ct}$ value was calculated by subtracting the WT $\Delta$ Ct value from $C d 37^{-1-} \Delta \mathrm{Ct}$ value. The fold change in the expression of each gene between WT and $C d 37^{-/}$mice was calculated as $2^{(-\Delta \Delta C t)}$. For quantitative PCR analysis of individual genes in $C d 37^{-/-}$tumor cells and WT control cells, RNA was isolated using the Quick-RNA Miniprep Kit (Zymo Research). RNA quantity and purity were determined on a NanoDrop spectrophotometer. RNA was treated with DNase I (amplification grade; Invitrogen) and reverse transcribed to cDNA by using random hexamers and Moloney murine leukemia virus reverse transcriptase (Invitrogen). mRNA levels of the genes of interest were determined with a CFX96 Sequence Detection System (Bio-Rad) with SYBR Green (Roche) as the fluorophore and gene-specific oligonucleotide primers. The primers used are as follows: Casp 8 (forward 5'-CAACTTCCTAGACTGCAACCG, reverse 5'-TCCAACTCGCTCACTTCTTCT), Ctnnb1 (forward 5'-CATCTTAAGCCCTCGCTCGG, reverse 5'-AGTAGCCATTGTCCACGCAG). Reaction mixtures and program conditions were used as recommended by the manufacturer (Bio-Rad). Quantitative PCR data were analyzed with the CFX Manager software (BioRad) and checked for correct amplification and dissociation of the products. Ct values of the genes of interest were normalized to the $\mathrm{Ct}$ value of the housekeeping gene Pbgd.

Determination of IL-6 levels. IL-6 levels in serum and supernatant were measured using standard ELISA procedures. Briefly, NUNC Maxisorp 96-well plates (eBioscience) were coated with capture antimouse IL-6 antibody (MP5-20F3, $2 \mu \mathrm{g} / \mathrm{ml}$, BD Pharmingen) in $0.1 \mathrm{M}$ carbonate buffer (pH 9.6) overnight at $4^{\circ} \mathrm{C}$. Wells were blocked with PBS containing 1\% BSA and 1\% FCS for 1 hour at room temperature, washed, and incubated with $50 \mu \mathrm{l}$ of sample and standard (2-fold serial dilutions starting from 10,000 pg/ml) (eBioscience). After 2-hour incubation at room temperature, wells were incubated with biotinylated anti-mouse IL-6 (MP5-32C11, $1 \mu \mathrm{g} / \mathrm{ml}$, BD Pharmingen) for 1 hour at room temperature, followed by incubation with HRP-conjugated streptavidin $(1: 5,000)$ for 30 minutes at room temperature. Complexes were visualized using TMB substrate (Sigma-Aldrich), and reaction was stopped by adding $0.8 \mathrm{M} \mathrm{H}_{2} \mathrm{SO}_{4}$. Absorbance was measured at $450 \mathrm{~nm}$ using an ELISA plate reader (iMark, Bio-Rad). IL-6 levels in sera of patients with DLBCL were measured using the Pelipair reagent set for human IL-6 (M9316, Sanquin) according to the manufacturer's instructions.

Cell culture, survival assays, and transfection. Freshly isolated Cd37-1tumor cells were cultured in RPMI 1640 (Invitrogen) containing 10\% FCS, $1 \%$ ultraglutamine, $1 \%$ antibiotic-antimycotic, $1 \mathrm{mM}$ sodium pyruvate, and $30 \mu \mathrm{M} \beta$-mercaptoethanol in the presence or absence of $10 \mu \mathrm{g} / \mathrm{ml}$ anti-mouse IL-6 (MP5-20F3, BD Pharmingen) or appropriate isotype control. For survival assays, viability of the neoplastic cells was assessed by trypan blue exclusion. The human B cell lymphoblastoid JY cell line, human pre-B cell line NALM-6, and HEK293 cells (all from ATCC) were cultured in RPMI 1640 (Invitrogen) supplemented with $10 \%$ FCS, $1 \%$ antibiotic-antimycotic, and $1 \%$ stable glutamine. Human CD37 construct (39) was cloned into psGFP2-N1 vector, placing the psGFP2 moiety on the C-terminus of the protein. NALM-6 cells were transiently transfected with human CD37-GFP using the SF Cell Line 4D-Nucleofector X Kit and 4D Nucleofector system (Lonza).

IL- 6 stimulation. Human NALM- 6 pre-B cells $\left(10 \times 10^{6}\right)$ or JY B cells $\left(2 \times 10^{6}\right)$ were stimulated with $15 \mathrm{ng} / \mathrm{ml}$ recombinant IL-6 (CellGro) for 60 or 75 minutes, respectively, at $37^{\circ} \mathrm{C}$ and $5 \% \mathrm{CO}_{2}$.

Determination of SOCS3 and p-STAT3 expression. Freshly isolated WT control and $C d 37^{-/}$tumor mLN cells $\left(10 \times 10^{6}\right)$ were lysed in 150 $\mathrm{mM} \mathrm{NaCl}, 10 \mathrm{mM}$ TrisHCl, $1 \%$ Triton X-100, $100 \mathrm{nM} \mathrm{NaVO}_{3}, 500 \mathrm{mM}$ NaF, 100 mM PMSF, and Complete Mini EDTA-free Protease Inhibitor Cocktail Tablet (Roche) and separated by reducing SDS-polyacrylamide gel electrophoresis. Western blots were incubated with goat 
anti-SOCS3 (M-20, 1:1,000, Santa Cruz Biotechnology) or rabbit antip-STAT3 (Tyr ${ }^{705}$, D3A7, 1:2,000, Cell Signaling Technology) and rabbit anti-actin (20-33, 1:1,000, Sigma-Aldrich) or rabbit anti-STAT3 (79D7, 1:2,000, Cell Signaling Technology) as loading control, followed by secondary IRDye anti-goat or anti-rabbit antibodies (LI-COR) and Odyssey infrared detection and Odyssey analysis software.

Coimmunoprecipitation experiments. Human CD37-EGFP (pEGFPN1) or empty pEGFP and SOCS3 (SIN-pgk) were transiently cotransfected into HEK293 cells using Metafectene (Biontex). After 16 hours, cells were lysed in lysis buffer (0.5\% NP40, $10 \mathrm{mM}$ TrisHCl pH 7.4, 150 $\mathrm{mM} \mathrm{NaCl}, 1 \mathrm{mM}$ EDTA, 1 mM Na $\mathrm{VO}_{4} \mathrm{pH}$ 10.0, 1 mM NaF, and 1x Protease Inhibitor Cocktail [Pharmingen]). Lysates were precleared with isotype control antibodies bound to protein G-Sepharose beads, followed by incubation with mouse anti-GFP (Roche), mouse anti-CD37 (WR17, made in-house; ref. 65), or mouse IgG (BioLegend) isotype control antibodies. After incubating for 2 hours at $4^{\circ} \mathrm{C}$, beads were washed 3 times in lysis buffer and SDS sample buffer containing 5\% $\beta$-mercaptoethanol was added. Samples were separated by SDS-PAGE, and Western blots were incubated with rabbit anti-GFP (made in-house) or rabbit anti-SOCS3 (Cell Signaling Technology, 2923) in TBS supplemented with $0.3 \%$ BSA and $0.1 \%$ skim milk powder at $4^{\circ} \mathrm{C}$ overnight and stained with secondary IRDye anti-rabbit antibodies (Invitrogen), followed by scanning membranes on the Odyssey Scanner (Westburg).

Copatching experiments. Human JY B cells were blocked with 3\% BSA, $10 \mathrm{mM}$ glycine, and $1 \%$ filtered human serum in PBS and stained with primary antibodies against human CD37 (WR17, made in-house; ref. 65), IL-6R $\alpha / C D 126$ (M5, BD), or isotype controls for 30 minutes at $4^{\circ} \mathrm{C}$. Secondary Alexa Fluor 488 or 647 antibodies (Invitrogen) were incubated for 30 minutes at $4^{\circ} \mathrm{C}$, followed by 1 hour at $12^{\circ} \mathrm{C}$. Cells were fixed with $2 \%$ PFA for 15 minutes at $4^{\circ} \mathrm{C}$, followed by 15 minutes at room temperature, and mounted on poly-L-lysine-coated glass slides at room temperature. For intracellular SOCS3 staining, the cell membrane was permeabilized with $0.5 \%$ saponin, $1 \%$ BSA, 10 mM glycine, and $1 \%$ filtered human serum in PBS, and cells were stained with antiSOCS3 (M-20, Santa Cruz Biotechnology) or isotype control for 45 minutes at room temperature, followed by secondary Alexa Fluor 488 or 647 antibodies (Invitrogen) for 30 minutes at room temperature. Glass slides were sealed with Mowiol (EMD Biosciences) on coverslips and imaged with an Olympus FV1000 Confocal Laser Scanning Microscope. Images were analyzed using Fiji software and Manders coefficients were determined using the JACoP plug-in.

Human IL6 and AKT1 mRNA expression. Gene expression analysis was performed on lymphoma tissues from patients with GCBDLBCL $(n=47)$, and mRNA expression of IL 6 and AKT1 was retrieved from the gene expression profiling data obtained from the Affymetrix GeneChip Human Genome HG-U133 Plus 2.0. The $\log _{2}$ expression values of the probe sets IL6 (205207_at) and AKT1 (207163_s_at) were used for analysis.

Statistics. Statistical differences in viability of $\mathrm{Cd} 37^{-/-}$tumor cells and between $\mathrm{CD}^{2} 7^{+}$and $\mathrm{CD} 37^{-}$groups were determined using unpaired Student's $t$ test (1 tailed) or nonparametric Mann-Whitney test (1 tailed, in case of non-Gaussian distribution). Statistical differences in IL-6 production in murine sera were determined using Kruskal-Wallis 1-way ANOVA test. OS was calculated from the time of diagnosis to death from any cause or last follow-up. PFS was calculated from the time of diagnosis to disease progression, relapse, or death from any cause. Patients who remained alive or progression free were censored at last follow-up. Survival analysis was performed using the Kaplan-Meier method with GraphPad Prism 5 (GraphPad Software), and differences were compared using the log-rank (Mantel-Cox) test. All differences were considered to be statistically significant at $P \leq 0.05$.

Study approval. All murine studies complied with European legislation (directive 2010/63/EU of the European Commision) and were approved by local authorities (CCD, The Hague, the Netherlands) for the care and use of animals with related codes of practice. Studies on human DLBCL tumor tissue were conducted in accordance with Declaration of Helsinki and were approved as being of minimal to no risk or as exempt by the Institutional Review Boards at The University of Texas MD Anderson Cancer Center.

\section{Author contributions}

ABVS designed the study. CMDW, SV, and ABVS wrote the manuscript. CMDW, SV, AVDS, and ISVH performed mouse experiments. MVDB, MJG, KMH, PJTAG, JHVK, and ABVS characterized the B cell lymphoma. CMDW, AVDS, and ABVS performed tissue stainings and in vitro tumor cell experiments. CMDW, SV, and AVDS did adoptive transfer experiments. CMDW and SV performed gene expression studies. CMDW and SE performed molecular studies of the IL-6 pathway. FAJVDL and MBB provided $I l 6^{-1-}$ mice and SOCS3 antibodies. KHY organized the lymphoma patient material. KHY, ZYXM, XW, YX, and KJJ performed the studies on human patients with lymphoma under approval of the IRB. CGF and ABVS supervised the work.

\section{Acknowledgments}

We thank Mark Wright (Monash University, Australia) for critical reading of the manuscript and Tessa van der Geest and Clive Michelo for their contribution to the research during their internship. We greatly appreciate the help from staff of the Central Animal Facility of the Radboud University Medical Center. A.B. van Spriel is supported by the Netherlands Organization for Scientific Research (NWO-ALW VIDI grant 864.11.006) and the Dutch Cancer Society (KUN2014-6845). K.H. Young is supported by The University of Texas MD Anderson Cancer Center Lymphoma Moon Shot Program, Institutional R\&D Fund, MD Anderson Cancer Center Lymphoma and Myeloma SPORE Research Development Program Awards, and the National Cancer Institute/NIH (R01CA138688, R01CA187415, P50CA136411, and P50CA142509). Z.Y. Xu-Monette is the recipient of the Shannon Timmins Leukemia Fellowship Award. C.G. Figdor is the recipient of an NWO Spinoza award, a European Research Council advanced grant PATHFINDER (269019), and a Koningin Wilhelmina Onderzoeksprijs award (KUN2009-4402) from the Dutch Cancer Society.

Address correspondence to: Annemiek B. van Spriel, Department of Tumor Immunology, Radboud Institute for Molecular Life Sciences, Radboud University Medical Center, Geert GrootepleinZuid 26-28, 6525GA Nijmegen, The Netherlands. Phone: 31.24.3617600; E-mail: Annemiek.vanSpriel@radboudumc.nl.

Sharon Veenbergen's present address is: Laboratory of Pediatrics, Division of Gastroenterology and Nutrition, Erasmus University Medical Center, Rotterdam, Netherlands. 
1. Shaffer AL, Rosenwald A, Staudt LM. Lymphoid malignancies: the dark side of B-cell differentiation. Nat Rev Immunol. 2002;2(12):920-932.

2. Weiss LM, Warnke RA, Sklar J, Cleary ML. Molecular analysis of the $\mathrm{t}(14 ; 18)$ chromosomal translocation in malignant lymphomas. $N$ Engl J Med.1987;317(19):1185-1189.

3. Visco C, et al. Patients with diffuse large B-cell lymphoma of germinal center origin with BCL2 translocations have poor outcome, irrespective of MYC status: a report from an International DLBCL rituximab-CHOP Consortium Program Study. Haematologica. 2013;98(2):255-263.

4. Limpens J, et al. Lymphoma-associated translocation $\mathrm{t}(14 ; 18)$ in blood $\mathrm{B}$ cells of normal individuals. Blood. 1995;85(9):2528-2536.

5. Summers KE, Goff LK, Wilson AG, Gupta RK, Lister TA, Fitzgibbon J. Frequency of the Bcl-2/ IgH rearrangement in normal individuals: implications for the monitoring of disease in patients with follicular lymphoma. JClin Oncol. 2001;19(2):420-424.

6. Rossi D, Ciardullo C, Gaidano G. Genetic aberrations of signaling pathways in lymphomagenesis: revelations from next generation sequencing studies. Semin Cancer Biol. 2013;23(6):422-430.

7. Shaffer AL 3rd, Young RM, Staudt LM. Pathogenesis of human B cell lymphomas. Annu Rev Immunol. 2012;30:565-610.

8. Burger R. Impact of interleukin-6 in hematological malignancies. Transfus Med Hemother. 2013;40(5):336-343.

9. Mihara M, Hashizume M, Yoshida H, Suzuki M, Shiina M. IL-6/IL-6 receptor system and its role in physiological and pathological conditions. Clin Sci (Lond). 2012;122(4):143-159.

10. Taga T, et al. Interleukin- 6 triggers the association of its receptor with a possible signal transducer, gp130. Cell. 1989;58(3):573-581.

11. Akira $\mathrm{S}$, et al. A nuclear factor for IL-6 expression (NF-IL6) is a member of a C/EBP family. ЕМВО J.1990;9(6):1897-1906.

12. Hideshima T, Nakamura N, Chauhan D, Anderson KC. Biologic sequelae of interleukin-6 induced PI3-K/Akt signaling in multiple myeloma. Oncogene. 2001;20(42):5991-6000.

13. Akira S, et al. Molecular cloning of APRF, a novel IFN-stimulated gene factor $3 \mathrm{p} 91$-related transcription factor involved in the gp130-mediated signaling pathway. Cell. 1994;77(1):63-71.

14. Naka T, et al. Structure and function of a new STAT-induced STAT inhibitor. Nature. 1997;387(6636):924-929.

15. Schmitz J, Weissenbach M, Haan S, Heinrich PC, Schaper F. SOCS3 exerts its inhibitory function on interleukin-6 signal transduction through the SHP2 recruitment site of gp130.J Biol Chem. 2000;275(17):12848-12856.

16. Nicholson SE, et al. Suppressor of cytokine signaling-3 preferentially binds to the SHP-2binding site on the shared cytokine receptor subunit gp130. Proc Natl Acad Sci U S A. 2000;97(12):6493-6498.

17. Babon JJ, et al. Suppression of cytokine signaling by SOCS3: characterization of the mode of inhibition and the basis of its specificity. Immunity. 2012;36(2):239-250.

18. Ding BB, et al. Constitutively activated STAT3 promotes cell proliferation and survival in the activated B-cell subtype of diffuse large B-cell lymphomas. Blood. 2008;111(3):1515-1523.

19. Sansone P, Bromberg J. Targeting the interleukin-6/Jak/stat pathway in human malignancies. JClin Oncol. 2012;30(9):1005-1014.

20. Voorzanger $\mathrm{N}$, et al. Interleukin (IL)-10 and IL- 6 are produced in vivo by non-Hodgkin's lymphoma cells and act as cooperative growth factors. Cancer Res. 1996;56(23):5499-5505.

21. Hemler ME. Tetraspanin functions and associated microdomains. Nat Rev Mol Cell Biol. 2005;6(10):801-811.

22. Levy S, Shoham T. The tetraspanin web modulates immune-signalling complexes. Nat Rev Immunol. 2005;5(2):136-148.

23. Charrin S, le Naour F, Silvie O, Milhiet PE, Boucheix C, Rubinstein E. Lateral organization of membrane proteins: tetraspanins spin their web. Biochem J. 2009;420(2):133-154.

24. Veenbergen S, van Spriel AB. Tetraspanins in the immune response against cancer. Immunol Lett. 2011;138(2):129-136.

25. Zhao X, et al. Targeting CD37-positive lymphoid malignancies with a novel engineered small modular immunopharmaceutical. Blood. 2007;110(7):2569-2577.

26. Heider KH, et al. A novel Fc-engineered monoclonal antibody to CD37 with enhanced ADCC and high proapoptotic activity for treatment of B-cell malignancies. Blood. 2011;118(15):4159-4168.

27. van Spriel AB, et al. The tetraspanin CD37 orchestrates the alpha(4)beta(1) integrin-Akt signaling axis and supports long-lived plasma cell survival. Sci Signal. 2012;5(250):ra82.

28. van Spriel AB, et al. The tetraspanin protein CD37 regulates IgA responses and anti-fungal immunity. PLoS Pathog. 2009;5(3):e1000338.

29. van Spriel AB, et al. A regulatory role for CD37 in T cell proliferation. J Immunol. 2004;172(5):2953-2961.

30. Sheng KC, et al. Tetraspanins CD37 and CD151 differentially regulate Ag presentation and T-cell co-stimulation by DC. Eur JImmunol. 2009;39(1):50-55.

31. Gartlan KH, et al. Tetraspanin CD37 contributes to the initiation of cellular immunity by promoting dendritic cell migration. Eur J Immunol. 2013;43(5):1208-1219.

32. Knobeloch KP, et al. Targeted inactivation of the tetraspanin CD37 impairs T-cell-dependent B-cell response under suboptimal costimulatory conditions. Mol Cell Biol. 2000;20(15):5363-5369.

33. Volk MJ, et al. Dietary restriction from middle age attenuates age-associated lymphoma development and interleukin 6 dysregulation in C57BL/6 mice. Cancer Res. 1994;54(11):3054-3061.

34. Morse HC 3rd, et al. Bethesda proposals for classification of lymphoid neoplasms in mice. Blood. 2002;100(1):246-258.

35. Ge X, Lv X, Feng L, Liu X, Wang X. High expression and nuclear localization of beta-catenin in diffuse large B-cell lymphoma. Mol Med Rep. 2012;5(6):1433-1437.

36. Fulda S. Caspase-8 in cancer biology and therapy. Cancer Lett. 2009;281(2):128-133.

37. Visco C, et al. Comprehensive gene expression profiling and immunohistochemical studies sup- port application of immunophenotypic algorithm for molecular subtype classification in diffuse large B-cell lymphoma: a report from the International DLBCL Rituximab-CHOP Consortium Program Study. Leukemia. 2012;26(9):2103-2113.

38. Naugler WE, Karin M. The wolf in sheep's clothing: the role of interleukin-6 in immunity, inflammation and cancer. Trends Mol Med. 2008;14(3):109-119.

39. Lapalombella R, et al. Tetraspanin CD37 directly mediates transduction of survival and apoptotic signals. Cancer Cell. 2012;21(5):694-708.

40. McDonnell TJ, et al. bcl-2-immunoglobulin transgenic mice demonstrate extended B cell survival and follicular lymphoproliferation. Cell. 1989;57(1):79-88.

41. Iqbal J, et al. BCL2 translocation defines a unique tumor subset within the germinal center B-celllike diffuse large B-cell lymphoma. Am J Pathol. 2004;165(1):159-166.

42. Kovalchuk AL, et al. IL-6 transgenic mouse model for extraosseous plasmacytoma. Proc Natl Acad Sci U S A. 2002;99(3):1509-1514.

43. Brocke-Heidrich K, et al. Interleukin-6dependent gene expression profiles in multiple myeloma INA- 6 cells reveal a Bcl-2 family-independent survival pathway closely associated with Stat3 activation. Blood. 2004;103(1):242-251.

44. Putoczki TL, et al. Interleukin-11 is the dominant IL-6 family cytokine during gastrointestinal tumorigenesis and can be targeted therapeutically. Cancer Cell. 2013;24(2):257-271.

45. Croker BA, et al. SOCS3 negatively regulates IL-6 signaling in vivo. Nat Immunol. 2003;4(6):540-545.

46. Inagaki-Ohara K, et al. Enhancement of leptin receptor signaling by SOCS3 deficiency induces development of gastric tumors in mice. Oncogene. 2014;33(1):74-84.

47. Wilop S, et al. Methylation-associated dysregulation of the suppressor of cytokine signaling-3 gene in multiple myeloma. Epigenetics. 2011;6(8):1047-1052.

48. Molavi O, Wang P, Zak Z, Gelebart P, Belch A, Lai R. Gene methylation and silencing of SOCS3 in mantle cell lymphoma. Br J Haematol. 2013;161(3):348-356.

49. Fojtova M, et al. Development of IFN-gamma resistance is associated with attenuation of SOCS genes induction and constitutive expression of SOCS 3 in melanoma cells. Br JCancer. 2007;97(2):231-237.

50. Nakagawa T, et al. Decreased expression of SOCS-3 mRNA in breast cancer with lymph node metastasis. Oncol Rep. 2008;19(1):33-39.

51. Attia FM, Hassan AM, El-Maraghy NN, Ibrahium $\mathrm{GH}$. Clinical significance of suppressor of cytokines signalling-3 mRNA expression from patients with non-Hodgkin lymphoma under chemotherapy. Cancer Biomark. 2011;11(1):41-47.

52. Hemler ME. Tetraspanin proteins promote multiple cancer stages. Nat Rev Cancer. 2014;14(1):49-60.

53. Luo RF, et al. CD81 protein is expressed at high levels in normal germinal center B cells and in subtypes of human lymphomas. Hum Pathol. 2010;41(2):271-280.

54. Yau JC, Dabbagh LK, Formenti KS, Coupland 


\section{RESEARCH ARTICLE}

RW, Burns BF, Shaw AR. Expression of transmembrane 4 superfamily member, $\mathrm{CD} 9$, is related to improved progression-free survival in patients with diffuse non-Hodgkin's lymphoma. Oncol Rep. 1998;5(6):1507-1511.

55. Jones PA, Laird PW. Cancer epigenetics comes of age. Nat Genet. 1999;21(2):163-167.

56. Yu W, et al. Epigenetic silencing of tumour suppressor gene $\mathrm{p} 15$ by its antisense RNA. Nature. 2008;451(7175):202-206.

57. Deckert J, et al. A novel anti-CD37 antibody-drug conjugate with multiple anti-tumor mechanisms for the treatment of B-cell malignancies. Blood. 2013;122(20):3500-3510.

58. Rafiq S, et al. Glycovariant anti-CD37 monospecific protein therapeutic exhibits enhanced effector cell-mediated cytotoxicity against chronic and acute B cell malignancies. MAbs. 2013;5(5):723-735.

59. Robak T, Robak P, Smolewski P. TRU-016, a humanized anti-CD37 IgG fusion protein for the potential treatment of B-cell malignancies. Curr Opin Investig Drugs. 2009;10(12):1383-1390.

60. Verstovsek S, et al. A double-blind, placebocontrolled trial of ruxolitinib for myelofibrosis. N Engl J Med. 2012;366(9):799-807.

61. Kopf M, et al. Impaired immune and acute-phase responses in interleukin-6-deficient mice. Nature. 1994;368(6469):339-342.

62. Ok CY, et al. Clinical implications of phosphorylated STAT3 expression in de novo diffuse large B-cell lymphoma. Clin Cancer Res. 2014;20(19):5113-5123.

63. van Dongen JJ, et al. Design and standardization of
PCR primers and protocols for detection of clonal immunoglobulin and T-cell receptor gene recombinations in suspect lymphoproliferations: report of the BIOMED-2 Concerted Action BMH4CT98-3936. Leukemia. 2003;17(12):2257-2317.

64. Langerak AW, et al. EuroClonality/BIOMED-2 guidelines for interpretation and reporting of Ig/ TCR clonality testing in suspected lymphoproliferations. Leukemia. 2012;26(10):2159-2171.

65. Schwartz-Albiez R, Dorken B, Hofmann W, Moldenhauer G. The B cell-associated CD37 antigen (gp40-52). Structure and subcellular expression of an extensively glycosylated glycoprotein. JImmunol. 1988;140(3):905-914.

66. Meyer-Wentrup F, et al. Dectin-1 interaction with tetraspanin CD37 inhibits IL-6 production. J Immunol. 2007;178(1):154-162. 